\title{
A Hybrid Forecasting Model Based on Bivariate Division and a Backpropagation Artificial Neural Network Optimized by Chaos Particle Swarm Optimization for Day-Ahead Electricity Price
}

\author{
Zhilong Wang, ${ }^{1}$ Feng Liu, ${ }^{2}$ Jie $W u,{ }^{2}$ and Jianzhou Wang ${ }^{2}$ \\ ${ }^{1}$ Department of Basic Courses, Lanzhou Institute of Technology, Lanzhou 730050, China \\ ${ }^{2}$ School of Mathematics and Statistics, Lanzhou University, Lanzhou 730000, China \\ Correspondence should be addressed to Feng Liu; liuf13@lzu.edu.cn
}

Received 2 April 2014; Accepted 19 May 2014; Published 14 July 2014

Academic Editor: Fuding Xie

Copyright (C) 2014 Zhilong Wang et al. This is an open access article distributed under the Creative Commons Attribution License, which permits unrestricted use, distribution, and reproduction in any medium, provided the original work is properly cited.

In the electricity market, the electricity price plays an inevitable role. Nevertheless, accurate price forecasting, a vital factor affecting both government regulatory agencies and public power companies, remains a huge challenge and a critical problem. Determining how to address the accurate forecasting problem becomes an even more significant task in an era in which electricity is increasingly important. Based on the chaos particle swarm optimization (CPSO), the backpropagation artificial neural network (BPANN), and the idea of bivariate division, this paper proposes a bivariate division BPANN (BD-BPANN) method and the CPSO-BDBPANN method for forecasting electricity price. The former method creatively transforms the electricity demand and price to be a new variable, named DV, which is calculated using the division principle, to forecast the day-ahead electricity by multiplying the forecasted values of the DVs and forecasted values of the demand. Next, to improve the accuracy of BD-BPANN, chaos particle swarm optimization and BD-BPANN are synthesized to form a novel model, CPSO-BD-BPANN. In this study, CPSO is utilized to optimize the initial parameters of BD-BPANN to make its output more stable than the original model. Finally, two forecasting strategies are proposed regarding different situations.

\section{Introduction}

The price of electricity is not only related to the interests of the market participants but also affected by many aspects of the relevant society and the economy. Thus, the price of electricity has been the subject of considerable research. Accurate forecasting of electricity price is of great significance, both for government regulatory agencies and for public power companies. However, the electricity prices exhibit large fluctuations, and the characteristics of the electricity prices in various electricity markets are notably different. Therefore, it is notably difficult to predict all of the electricity prices using only one model. As indicated by Anbazhagan and Kumarappan, in general, the forecasting errors for the electricity price vary from approximately $5 \%$ to $36 \%$, which are relatively high compared with the forecasting errors for the electricity load (usually in the range from $1 \%$ to $3 \%$ ). Thus, there is still an urgent demand for exploiting new electricity price forecasting models to improve the electricity price forecasting accuracy [1].

Existing forecasting models for the electricity price can be categorized by different classification criteria. Two criteria are adopted frequently, that is, forecasting models based classification and forecasting horizons based classification. According to the former classification criterion, the electricity price forecasting models can be generally classified into three categories [2]: (i) the stochastic models, (ii) the causal forecasting approaches, and (iii) the artificial intelligence based methods. According to the latter criterion, forecasting models for electricity price consist of three types: the shortterm, the midterm, and the long-term models. However, as is known, most forecasting models cannot be sorted into 
a single category by the forecasting models based classification rule because increasing numbers of hybrid or combined models are being developed to obtain electricity price results with superior performance. Therefore, this paper will survey and summarize the previous electricity price forecasting models according to their forecasting horizons. The shortterm electricity price forecasting is usually known as the day-ahead electricity price forecasting. During the recent few decades, a large number of day-ahead electricity price forecasting models have been exploited for their importance in establishing bidding strategies for the spot market [3]. A two-stage model derived from panel cointegration and a particle filter was proposed by $\mathrm{Li}$ et al. [4] to forecast the day-ahead electricity data of Pennsylvania, New Jersey, Maryland (PJM). Zhang et al. [5] presented the performance of a hybrid approach composed of the wavelet transform (WT) method, the autoregressive integrated moving average (ARIMA) model, the least squares support vector machine (LSSVM), and the particle swarm optimization in forecasting the day-ahead electricity price of New South Wales, Australia. The WT and ARIMA algorithms were also used by Tan et al. [6] to build a hybrid model with the generalized autoregressive conditional heteroskedasticity to examine the day-ahead electricity price forecasting performance of PJM and Spain. A day-ahead price forecasting model, which was constructed by a feature selection approach and a cascaded neural network algorithm, was developed by Amjady and Keynia [7] to perform an electricity price survey in Spain and Australia. Several other day-ahead electricity price forecasting models can be found in [8-15]. Accurate midterm electricity price forecasting is essential in assisting the market players to define their contracts and hedging policies. Horizons for midterm electricity price forecasting usually last from one month ahead to six months ahead. Yan and Chowdhury [16] presented a hybrid forecasting model of the midterm electricity clearing price on the basis of the LSSVM and autoregressive moving average with external input models. A multiple support vector machine approach was also applied by the same two authors [17] to the electricity market clearing price forecasting by using the PJM interconnection data. Compared to the short-term and midterm electricity price forecasting tasks, the long-term electricity price forecasting is conducted on a much wider horizon to provide the market players with the ability to make expansion plans. Pao [3] adopted an artificial neural network model to survey the long-term electricity price forecasting results on the European Energy Exchange market. However, studies regarding the long-term electricity price forecasting are relatively few compared to the number of studies on short-term and midterm electricity price forecasting due to the relatively long forecasting horizons required.

The accuracy of electricity price forecasting is affected by many factors, such as historical price, temperature, and load, among which the effect of load on electricity price forecasting is quite significant. Much of the relevant literature attempted to improve the accuracy of the electricity price by considering the impact of electricity load on the electricity price. For example, Lin et al. [18] regarded the load as one of the inputs to the enhanced probability neural network proposed in their paper to demonstrate its performance in the electricity price forecasting. Load was also contained in the input variables of the enhanced radial basis function network proposed by Lin et al. [15]. Singhal and Swarup [19] also used the historical load and forecasted load as inputs to the artificial neural network models to obtain the electricity price forecasting results. Although the specific models used in these studies are different, these studies have one factor in common: the impact of the load on the electricity price in these studies was embodied by directly using the load as one of the input variables and the electricity price as the output variable, rather than to transform both electricity demand and price into a new variable. Based on this idea of transformation, a new hybrid electricity model is proposed in this paper.

\section{Backpropagation Artificial Neuron Network (BPANN)}

An artificial neural network is widely used to validate the underlying relationship between the relevant dependent and independent variables. The advantage of the use of neural networks compared to the traditional methods lies in their capacity to analyze complicated patterns quickly with a low error. In addition, in neural networks, no assumptions are required regarding the characteristics of the underlying distribution of the data [20]. Among all of the neural networks, the backpropagation artificial neural network (BPANN) is the most frequently used neural network. The basic BPANN consists of three parts: the input layer part, the hidden layer part (or middle layer part, as one or more layers can be contained in this part), and the output layer part. Generally, the number of neurons in the input layer and in the output layer is determined according to the desired input variables and output variables, respectively, while there is no fixed rule to choose the number of the neurons in the hidden layer part. For a BPANN with $N$ neurons $x_{1}, x_{2}, \ldots, x_{N}$ in the current layer, the output $y_{k}$ of the $k$ th neuron in the adjacent next layer is expressed as

$$
y_{k}=f\left(\sum_{i=1}^{N} w_{i k} x_{i}-b_{k}\right),
$$

where $w_{i k}$ is the weight connection from the neuron $i$ of the current layer to the neuron $k$ of the adjacent next layer, $b_{k}$ is the threshold of the $k$ th neuron, and $f(\cdot)$ is the activation function, which usually consists of three types [21]:

(i) $f(\cdot)$ is the linear function defined as

$$
f(x)=x ;
$$

(ii) $f(\cdot)$ is the logistic sigmoid (logsig) function defined as

$$
f(x)=\frac{1}{1+\exp (-x)}
$$

(iii) $f(\cdot)$ is the hyperbolic sigmoid (tansig) function defined as

$$
f(x)=\frac{2}{1+\exp (-2 x)}-1 .
$$


To validate the performance of a $\mathrm{BP}$ neural network, the sample data are usually divided into three parts: the training data, the validation data, and the testing data. If there are $M$ input-output pairs in the training data in total and the number of the neurons in the output layer is $K$, then the objective function of the BPANN is given by

$$
E=\frac{1}{2 M} \sum_{i=1}^{M} \sum_{j=1}^{K}\left(\mathrm{do}_{j}^{i}-\mathrm{ao}_{j}^{i}\right)^{2},
$$

where $\mathrm{do}_{j}^{i}$ and $\mathrm{ao}_{j}^{i}$ are the desired and actual outputs, respectively, of the $j$ th neuron obtained by the $i$ th input-output pair.

In the operation process of the BPANN, the weight is updated according to the gradient descent rules expressed as follows [22]:

$$
\begin{aligned}
& b_{k}(i+1)=b_{k}(i)-\frac{\eta \partial E}{\partial b_{k}} \\
& w_{i k}(i+1)=w_{i k}(i)-\frac{\eta \partial E}{\partial w_{i k}}
\end{aligned}
$$

where $\eta$ is the learning rate parameter. In this paper, BPANN has three layers, the weights and thresholds of which are denoted by $w_{1}, b_{1}$ and $w_{2}, b_{2}$. The activation function of first layer is a logistic sigmoid function, and that of the second layer is a linear function.

\section{Bivariate Division BPANN (BD-BPANN)}

In Section 1, it is mentioned that electricity price and demand have a closed relationship. In addition, Singhal and Swarup obtained the result that the price forecasting values using the neural network model indicate that the electricity price in the deregulated markets is strongly dependent on the trend in the load demand and the clearing price [19]. Therefore, to promote the accuracy of price forecasting, a bivariate division model based on BPANN, the price databases, and the demand databases is proposed. This hybrid approach skillfully transforms both of the two variables, demand and price, into one, as denoted by DV, using the dividing principle. This principle can be shown as

$$
\mathrm{DV}=\frac{\text { Demand }}{\text { Price }} \text {. }
$$

Next, a sample matrix of DV is used to obtain the parameters of BPANN, which will provide the forecasting values of DV. Meanwhile, the network of electricity demand is also obtained by training its BPANN. Eventually, the electricity price forecasting values can be calculated using the following simple formula:

$$
\text { Price }_{\text {forecasting }}=\frac{\text { Demand }_{\text {forecasting }}}{\mathrm{DV}_{\text {forecasting }}} .
$$

Details of BD-BPANN can be shown by these steps.

Step 1. Obtain the values of DV by using DV = Demand/ Price.
Step 2. Perform preprocessing of the training set of electricity demand using the following formula:

$$
\begin{aligned}
& \text { Data }_{\text {Training Set }} \\
& =\frac{\text { Data }_{\text {Training Set }}-\min \left(\text { Data }_{\text {Training Set }}\right)}{\max \left(\text { Data }_{\text {Training Set }}\right)-\min \left(\text { Data }_{\text {Training Set }}\right)} .
\end{aligned}
$$

In addition, the testing set will be mapped by

$$
\operatorname{Data}_{\text {Testing Set }}=\frac{\text { Data }_{\text {Testing Set }}-\min \left(\text { Data }_{\text {Training Set }}\right)}{\max \left(\text { Data }_{\text {Training Set }}\right)-\min \left(\text { Data }_{\text {Training Set }}\right)} .
$$

Note that the inputs and the outputs of the training sets and testing sets themselves are column vectors.

Step 3. Use Data ${ }_{\text {Training Set }}$ to train the BPANN and obtain BPANN ${ }_{\text {Demand }}$.

Step 4. Obtain the mapping values of the inputs of Data $_{\text {Testing Set }}$ through

$$
\begin{aligned}
& \text { Output }_{\text {BPANN }_{\text {Demand }}} \\
& =\text { BPANN }_{\text {Demand }} \text { (inputs of } \\
& \text { the demand part of Data }{ }_{\text {Testing Set }} \text { ). }
\end{aligned}
$$

Step 5. Obtain the forecasting values by postprocessing of the output by

$$
\begin{aligned}
& \text { Demand }_{\text {Forecasting }} \\
& =\text { Output }_{\text {BPANN }_{\text {Demand }}} \times\left(\max \left(\text { Data }_{\text {Training Set }}\right)\right. \\
& \left.-\min \left(\text { Data }_{\text {Training Set }}\right)\right) \\
& +\min \left(\text { Data }_{\text {Training Set }}\right) \text {. }
\end{aligned}
$$

Step 6. Replace the electricity demand with DV values and repeat Steps 2-5 to obtain the forecasting values of the DVs.

Step 7. Obtain the forecasting price by

$$
\text { Price }_{\text {Forecasting }}=\frac{\text { Demand }_{\text {Forecasting }}}{\mathrm{DV}_{\text {Forecasting }}} \text {. }
$$

Figure 1 shows the specific process of BD-BPANN.

\section{Chaos Particle Swarm Optimization BD-BPANN (CPSO-BD-BPANN)}

The particle swarm optimization (PSO) algorithm was first developed by Kennedy and Eberhart [23] as a populationbased optimization approach. In a PSO system, there are multiple candidate solutions, and each solution is named a "particle." Each particle is captured by two factors: the velocity and the position. 


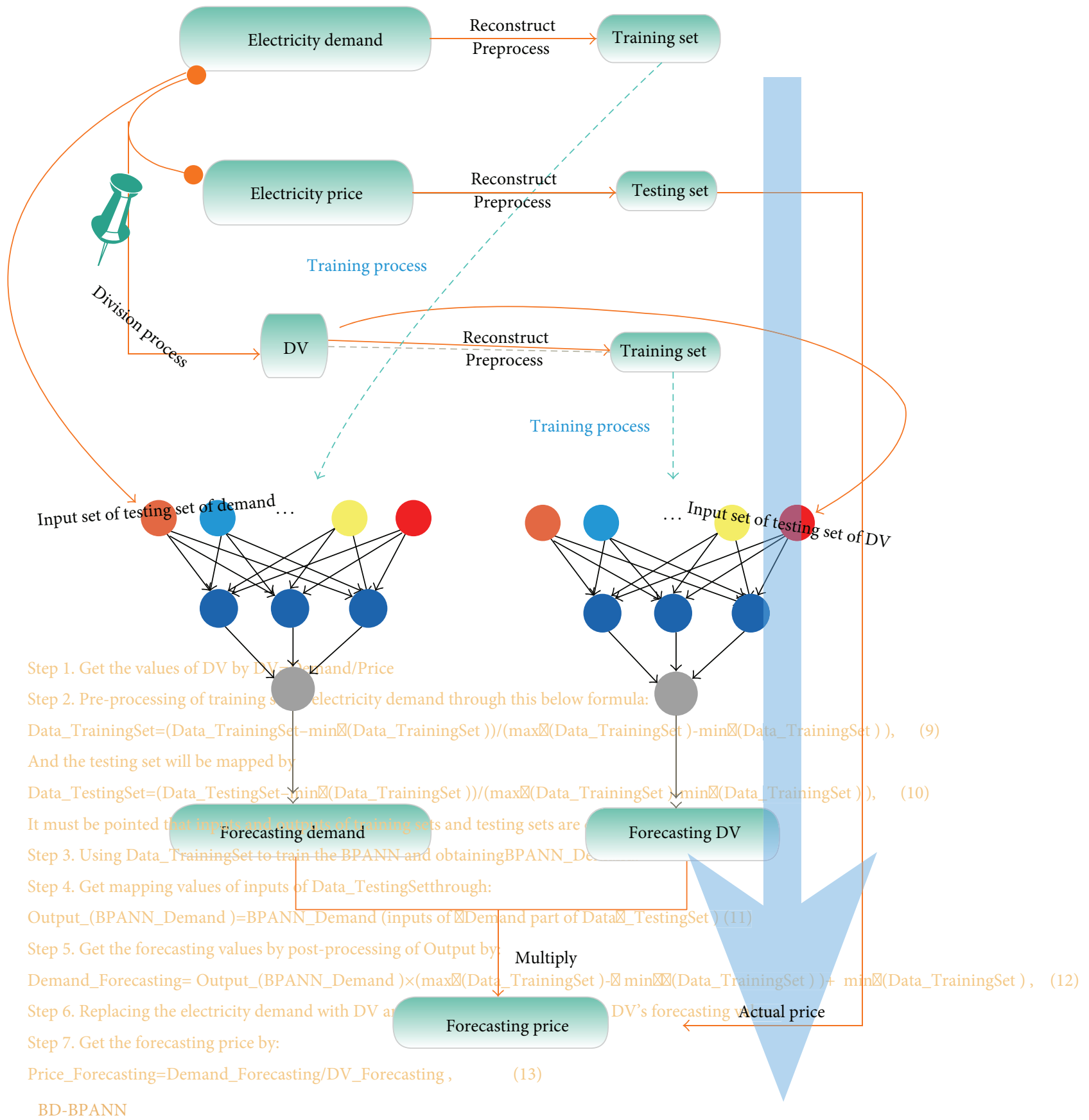

Figure 1: BD-BPANN method.

4.1. Chaos Particle Swarm Optimization (CPSO). In a standard PSO, at iteration $t+1$, for the $k$ th particle, its velocity $\left(v_{k}\right)$ and the position $\left(x_{k}\right)$ are updated by [24]

$$
\begin{gathered}
v_{k}^{t+1}=w v_{k}^{t}+c_{1} \times \operatorname{rand} 1 \times\left(P_{k}-x_{k}^{t}\right)+c_{2} \\
\times \operatorname{rand} 2 \times\left(P_{g}-x_{k}^{t}\right), \\
x_{k}^{t+1}=x_{k}^{t}+v_{k}^{t+1},
\end{gathered}
$$

where $w$ is the inertia weight, rand1 and rand2 are two independent random variables drawn from a uniform distribution with range $[0,1]$, and $c_{1}$ and $c_{2}$ are positive constant parameters that are known as the learning parameters. The remaining two variables $P_{k}$ and $P_{g}$ denote the personal best position of the $k$ th particle in history and the current global best position, respectively; that is, by substituting these two values into the predetermined objective function, the optimal values of the $k$ th particle and the whole particles can be obtained. 
Although the standard PSO is widely applied in the parameter optimization field, it had disadvantages of easily remaining in the local optimization of the parameters, and its convergence speed is slow. As indicated by Singhal and Swarup [19], the use of the chaos will accelerate the convergence speed due to the nonrepetitive nature of chaos. Thus, the chaotic PSO (CPSO) algorithm is adopted in this paper to obtain the optimal parameter values with a higher convergence speed. The improvement of the CPSO algorithm over the standard PSO is that it initializes the position of the particles by the following formula:

$$
x_{i+1}=\mu x_{i}\left(1-x_{i}\right), \quad i=1,2, \ldots, n,
$$

where $\mu$ is the control variable located in the range $(2,4]$.

4.2. CPSO-BD-BPANN. In the classical training process of an artificial neuron network, especially for that of BPANN, the initial parameters, $w_{1}$ and $w_{2}$ along with $b_{1}$ and $b_{2}$, often, are random matrices or vectors and consequently easily fall into the local optimal solution [25]. Chaos particle swarm optimization (abbreviated as CPSO) is an effective artificial algorithm that has the ability to achieve global optimization. CPSO is based on the basic concept of particle swarm optimization and the theory of chaos. In this paper, CPSO is used to achieve the best initial parameters of the BPANN. The improved network outperforms the previous one and has an improved ability to determine the global optimal parameters, with a lower probability of overfitting and a higher forecasting accuracy. Main steps of this CPSO-BPANN are described as follows.

Step 1. Reshape the parameters $w_{1}, w_{2}$ and $b_{1}, b_{2}$ of the BPANN to one vector and denote the length of the vector by $n$.

Step 2. Perform the initialization of the CPSO's swarm, whose length is $n$.

Step 3. Choose the fitness evaluation of CPSO to be the mean squared error (MSE):

$$
\text { MSE }=\frac{\left(\text { Vector }_{\text {Actual }}-\text { Vector }_{\text {Network Output }}\right)^{2}}{\text { Length }_{\text {Vector }_{\text {Actual }}}} .
$$

Step 4. Execute the optimizing process of the CPSO and obtain the best swarm.

Step 5. Reshape the best swarm into four parameters of the network, and then reconstruct the BPANN by using these optimal matrices and vectors. The resulting network is called CPSO-BPANN.

Next, the BD-BPANN will replace the BPANN's part of the CPSO-BPANN. The process of the new forecasting model is as follows.

Step 1. Obtain the values of DV by using DV = Demand/ Price.
Step 2. Perform the preprocessing of the training set of the electricity demand using the following formula:

$$
\begin{aligned}
& \text { Data }_{\text {Training Set }} \\
& =\frac{\text { Data }_{\text {Training Set }}-\min \left(\text { Data }_{\text {Training Set }}\right)}{\max \left(\text { Data }_{\text {Training Set }}\right)-\min \left(\text { Data }_{\text {Training Set }}\right)} .
\end{aligned}
$$

And the testing set will be mapped by

$$
\text { Data }_{\text {Testing Set }}=\frac{\text { Data }_{\text {Testing Set }}-\min \left(\text { Data }_{\text {Training Set }}\right)}{\max \left(\text { Data }_{\text {Training Set }}\right)-\min \left(\text { Data }_{\text {Training Set }}\right)} .
$$

Note that the inputs and outputs of the training sets and the testing sets are column vectors.

Step 3. Construct the BPANN based on the structure of the data.

Step 4. Reshape the parameters, $w_{1}, w_{2}$ and $b_{1}, b_{2}$, of the BPANN into one vector, and denote the length of the vector as $n$.

Step 5. Perform the initialization of the CPSO's swarm, whose length is $n$.

Step 6. Choose the fitness evaluation of CPSO as the mean squared error (MSE):

$$
\text { MSE }=\frac{\left(\text { Vector }_{\text {Actual }}-\text { Vector }_{\text {Network Output }}\right)^{2}}{\text { Length }_{\text {Vector }_{\text {Actual }}}} .
$$

Step 7. Execute the optimization process of the CPSO and obtain the best swarm.

Step 8. Reshape the best swarm into four parameters of the network, and then reconstruct the BPANN, consequently named as CPSO-BPANN, by using these optimal matrices and vectors.

Step 9. Use Data ${ }_{\text {Trainingset }}$ to train the CPSO-BPANN to obtain CPSO-BPANN Demand $_{\text {. }}$

Step 10. Obtain the mapping values of the inputs of Data $_{\text {Testing Set }}$ through

Output $_{\text {CPSO-BPANN }}$ Demand

$=\mathrm{CPSO}_{\mathrm{BPANN}} \mathrm{Demand}_{\text {(inputs of the demand }}$

$$
\text { part of Data } \operatorname{Testing~Set~}) \text {. }
$$


Step 11. Obtain the forecasting values by postprocessing of the output by

$$
\begin{aligned}
& \text { Demand }_{\text {Forecasting }} \\
& =\text { Output }_{\text {CPSO-BPANN }}{ }_{\text {Demand }} \\
& \quad *\left(\max \left(\text { Data }_{\text {Training Set }}\right)-\min \left(\text { Data }_{\text {Training Set }}\right)\right) \\
& \quad+\min \left(\text { Data }_{\text {Training Set }}\right) .
\end{aligned}
$$

Step 12. Replace the electricity demand with DV and repeat Steps 2-11 to obtain the forecasted values of the DVs.

Step 13. Obtain the forecasted price by

$$
\text { Price }_{\text {Forecasting }}=\frac{\text { Demand }_{\text {Forecasting }}}{\mathrm{DV}_{\text {Forecasting }}} .
$$

Figure 2 schematically shows the entire process of the new model.

\section{Numerical Results}

In this section, the electricity price and demand will be used to test the models of CPSO-BD-BPANN, BD-BPANN, CPSO-BPANN, and BPANN. To begin, the source of data, the forecasting principle, and the cases that must be studied are introduced. Next, cases 1 and 2 are researched specifically with data provided in tables and figures. Finally, the remaining cases are studied, and, consequently, evaluation of the models is performed by comparing the forecasting accuracy, calculation time, and stability.

5.1. Data Selection, Forecasting Principles, and Case Studies. In Data Selection section, the source of the electricity data and certain properties of the data will be briefly introduced. Because a comparison is performed among these models, it is necessary to establish certain principles, which will be mentioned in the section Forecasting Principles. Eventually, to obtain the overall forecasting effectiveness of each model, this paper chooses five cases to illustrate the overall forecasting results calculated by the four models considered.

5.1.1. Data Selection. The electricity price and demand of Victoria of Australia in 2008 are chosen as the database under consideration in this paper. In each day of this database, there are 48 observation points, each representing half-hours. Figure 3 shows the schematic diagram of the electricity price, and Figure 4 shows the schematic diagram of the demand.

It is obvious that demand data are more regular than the electricity price data.

\subsubsection{Forecasting Principles}

Principle 1. This new forecasting model, whose lifespan is one day, will be updated by reconstructing and training new samples when it has provided 48 forecasting values.
Principle 2. The four-hour points will be used to forecast the price of the next half-hour.

Principle 3. The number of neurons in the hidden layer of the CPSO-BPANN is 3.

Principle 4. The points of the previous 21 days of the target day, a total of 1008 half-hour values, construct the training set.

Principle 5. In this paper, the forecasting result of the BPANN is the best result among the 10 times the forecasting results are obtained; this best BPANN is abbreviated as b-BPANN.

Principles 1-4 are schematically shown in Figure 5.

5.1.3. Study Cases. There are five cases in this paper. The purpose of case 1 is to explain the proposed model by forecasting only one day specifically. The other cases concentrate on the forecasting effectiveness of the proposed model and a comparison among the new CPSO-BD-BPANN and CPSO$\mathrm{BPANN}$ and the original b-BPANN. The evaluation criterion of the models is mean absolute percent error (MAPE), which can be expressed as

$$
\text { MAPE }=\frac{\left|y_{\text {models }}-y_{\text {actual }}\right|}{y_{\text {actual }}} \times 100 \% .
$$

And it must be pointed out that elements of all columns except the first column are MAPEs which is dimensionless in Tables 4, 5, 6, 7, and 8. The details of each case are presented in Table 1 .

5.2. Study of Case 1. In this case, each step, as mentioned in Section 4, will be clearly presented through figures and tables to understand the main concept of this proposed model. First, electricity price, demand, and the value of DV (as described in Section 3) are presented in Figure 6.

The electricity demand clearly has more regularity in the data than those of price and DV. In addition, the fact that the volatility of price and DV do not exhibit distinct differences is also observed. Next, the results of the BD-BPANN will be presented in Table 2 and Figure 7.

In Table 2, units of demand and price are $\mathrm{Hmk} / \mathrm{h}$ and $\$ /$ Mwh, respectively. The symbol “*” represents the time spots, a total of 27 points, for which BD-BPANN outperforms b-BPANN. The symbol " $\dagger$ " represents the reverse situation to that of the symbol “*." The symbol “ $\ddagger$ ” represents the results of the average and the variance of each column (except the first column). Specifically, in these 48 time spots, the best forecasting MAPE of demand is $0.05 \%$ at $10: 30$ of this day, and the worst MAPE is $6.06 \%$ at $1: 00$. The average value, the variance, and average forecasting MAPE of the electricity demand obtained through b-BPANN are 5645.0323, 555.27757, and 1.38\%, respectively. Similarly, the best forecasting value of the DV whose MAPE is $1.25 \%$ is at 11:30 of June 9 in 2008, and the worst performance of forecasting occurred at 2:30. The average value, variance, and average forecasting MAPE of the DV which b-BPANN calculates are $222.68,88.982$, and $11.24 \%$, respectively. To 


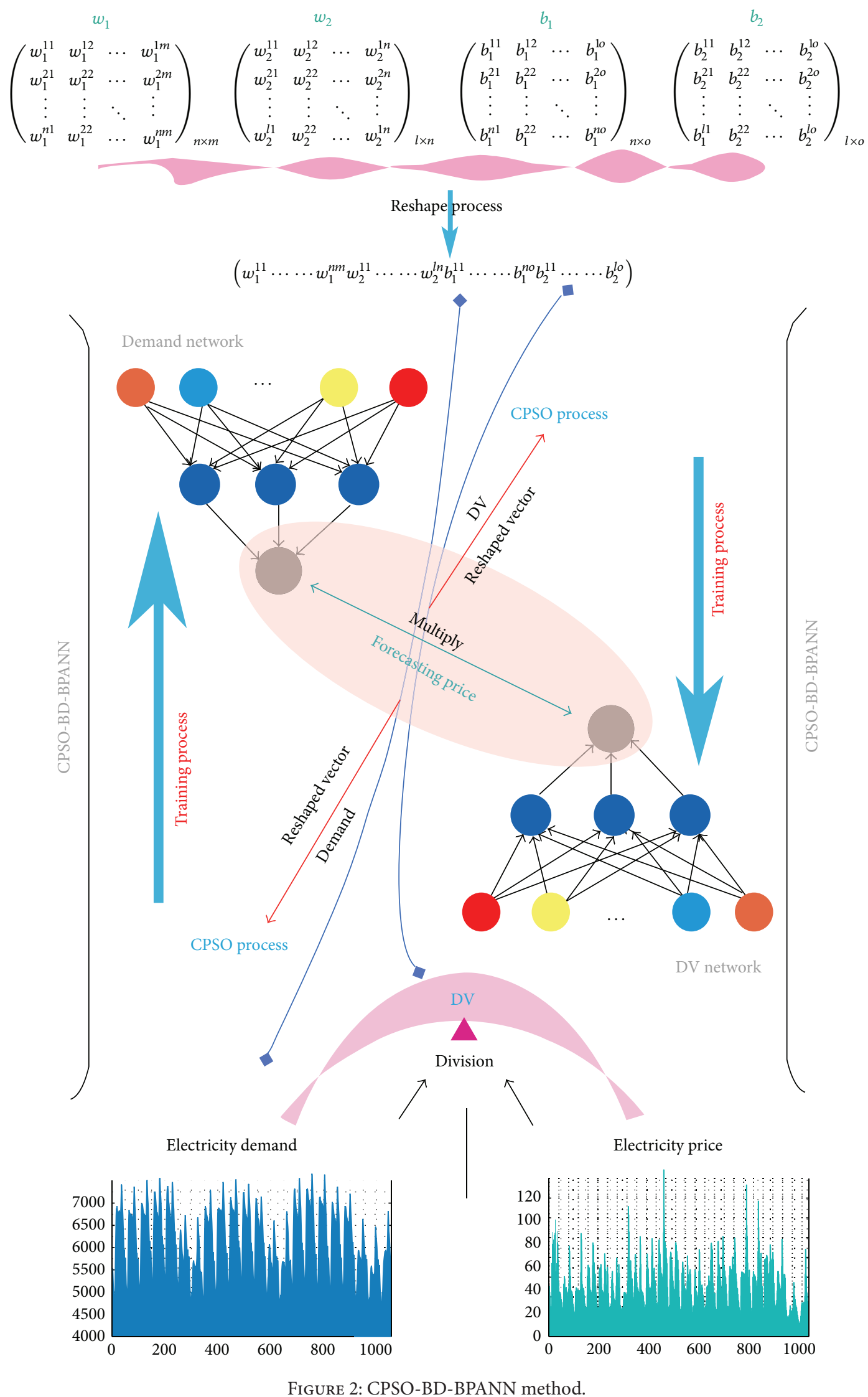


TABLE 1: Cases' information.

\begin{tabular}{|c|c|c|c|c|}
\hline Case & Testing days & Sample (training) days & Numbers of samples & Marks \\
\hline 1 & June 9, 2008 & May 19-June 8, 2008 & 1008 & Example \\
\hline \multirow{7}{*}{2} & March 10, 2008 & February 18-March 9, 2008 & 1008 & \multirow{7}{*}{ Spring } \\
\hline & March 11, 2008 & February 19-March 10, 2008 & 1008 & \\
\hline & March 12, 2008 & February 20-March 11, 2008 & 1008 & \\
\hline & March 13, 2008 & February 21-March 12, 2008 & 1008 & \\
\hline & March 14, 2008 & February 22-March 13, 2008 & 1008 & \\
\hline & March 15, 2008 & February 23-March 14, 2008 & 1008 & \\
\hline & March 16, 2008 & February 24-March 15, 2008 & 1008 & \\
\hline \multirow{7}{*}{3} & June 9, 2008 & May 19-June 8, 2008 & 1008 & \multirow{7}{*}{ Summer } \\
\hline & June 10, 2008 & May 20-June 9, 2008 & 1008 & \\
\hline & June 11, 2008 & May 21-June 10th, 2008 & 1008 & \\
\hline & June 12, 2008 & May 22-June 11, 2008 & 1008 & \\
\hline & June 13, 2008 & May 23-June 12, 2008 & 1008 & \\
\hline & June 14, 2008 & May 24-June 13, 2008 & 1008 & \\
\hline & June 15, 2008 & May 25-June 14, 2008 & 1008 & \\
\hline \multirow{7}{*}{4} & September 15, 2008 & August 25-September 14, 2008 & 1008 & \multirow{7}{*}{ Autumn } \\
\hline & September 16, 2008 & August 26-September 15, 2008 & 1008 & \\
\hline & September 17, 2008 & August 27-September 16, 2008 & 1008 & \\
\hline & September 18, 2008 & August 28-September 17, 2008 & 1008 & \\
\hline & September 19, 2008 & August 29-September 18, 2008 & 1008 & \\
\hline & September 20, 2008 & August 30-September 19, 2008 & 1008 & \\
\hline & September 21, 2008 & August 31-September 20, 2008 & 1008 & \\
\hline \multirow{7}{*}{5} & December 8, 2008 & November 17-December 7, 2008 & 1008 & \multirow{7}{*}{ Winter } \\
\hline & December 9, 2008 & November 18-December 8, 2008 & 1008 & \\
\hline & December 10, 2008 & November 19-December 9, 2008 & 1008 & \\
\hline & December 11, 2008 & November 20-December 10, 2008 & 1008 & \\
\hline & December 12, 2008 & November 21-December 11, 2008 & 1008 & \\
\hline & December 13, 2008 & November 22-December 12, 2008 & 1008 & \\
\hline & December 14, 2008 & November 23-December 13, 2008 & 1008 & \\
\hline
\end{tabular}

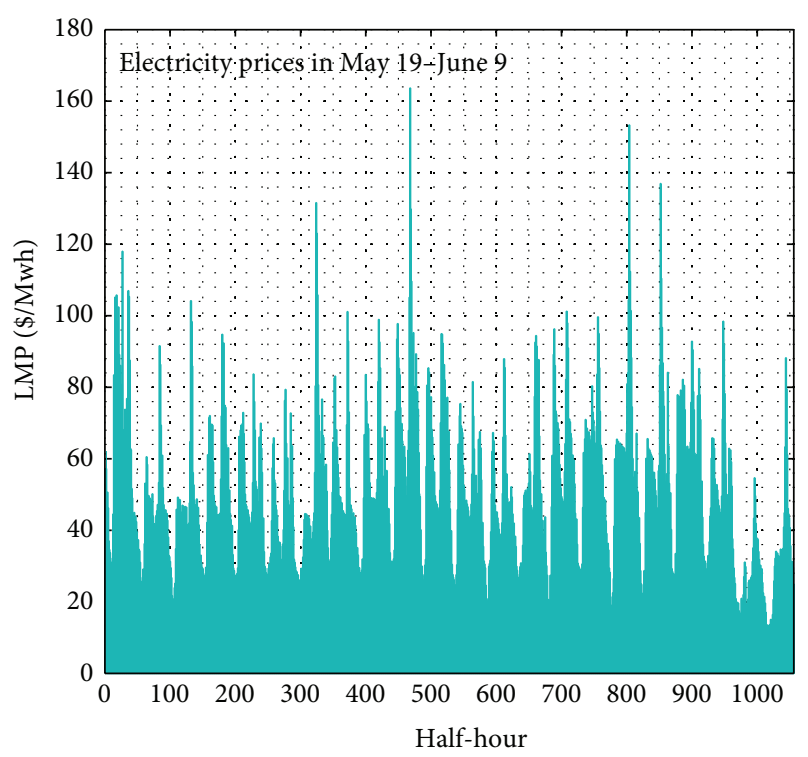

FIGURE 3: Electricity prices in the period from May 19 to June 9.

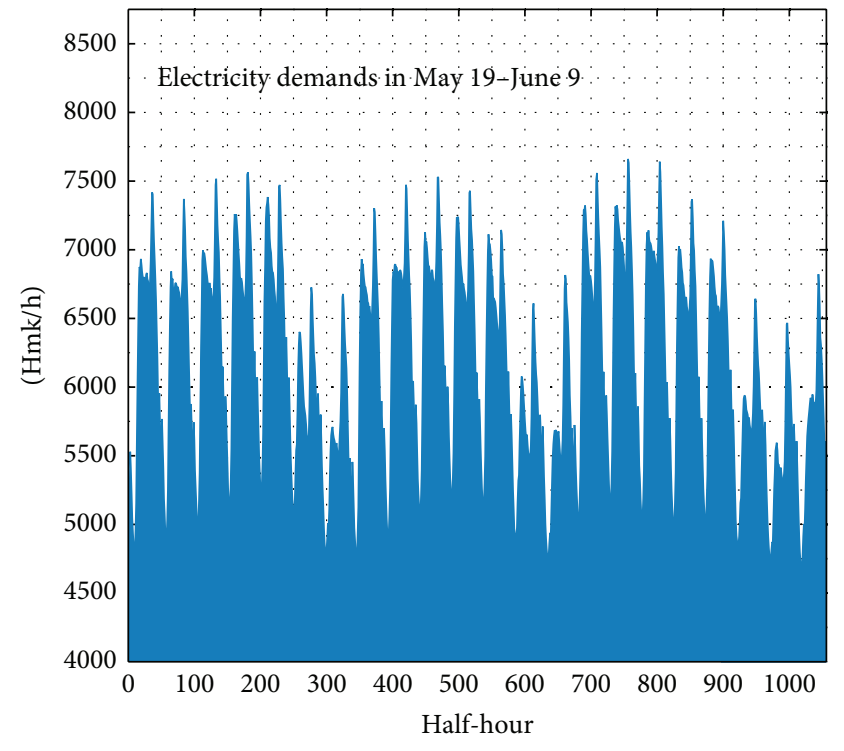

FIGURE 4: Electricity demand in the period from May 19 to June 9. 


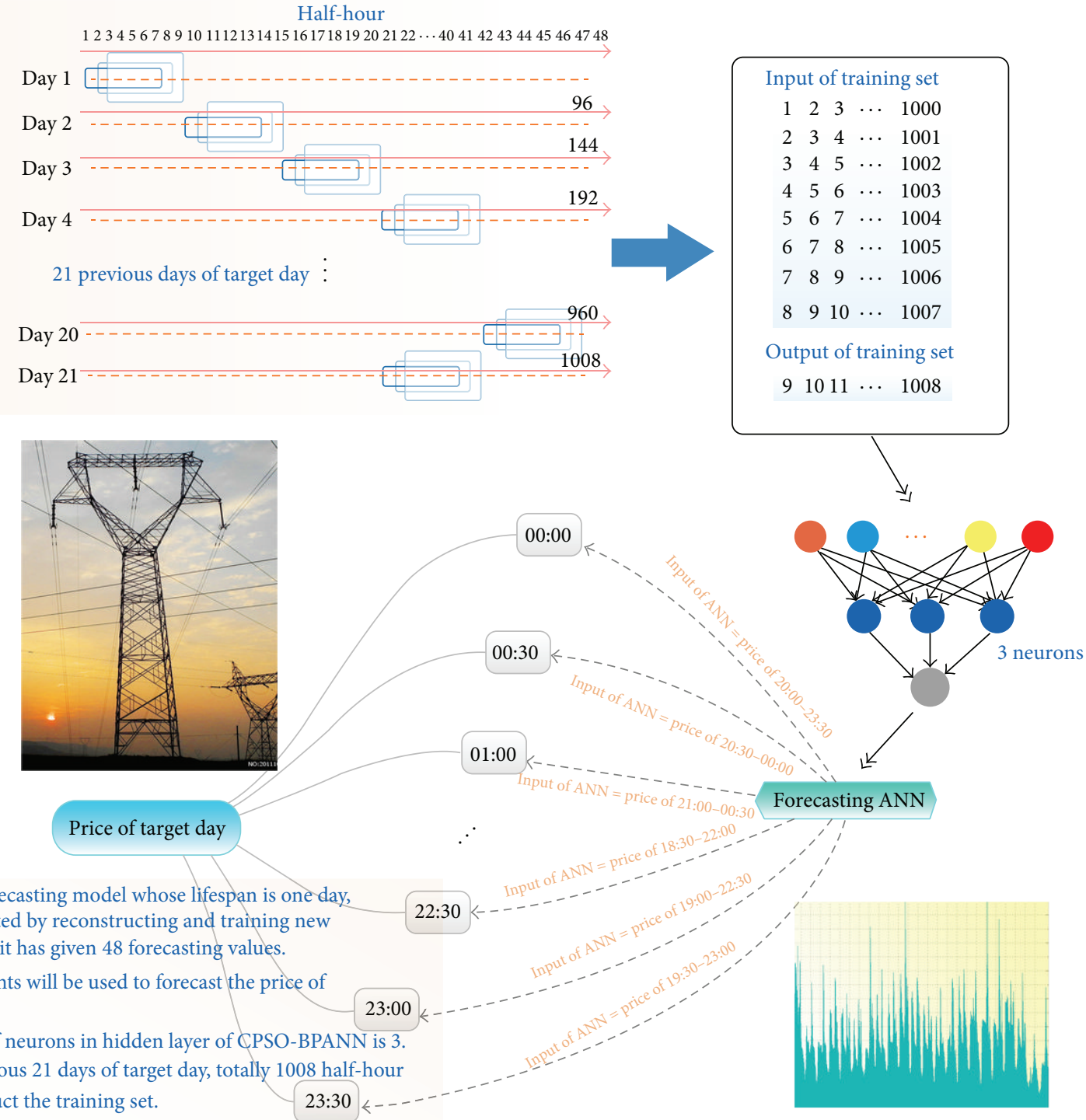

Figure 5: Forecasting Principles 1-4.

make a comparison between b-BPANN and BD-BPANN, the output of b-BPANN, that is, the price forecasting values obtained by only using b-BPANN, is also presented in Table 2. $0.52 \%$ is the best MAPE of b-BPANN at $16: 30$, and $42.39 \%$ is the worst MAPE obtained when utilizing b-BPANN to forecast electricity price occurred at 2:30. In addition, the average value, variance, and average forecasting MAPE of the price are $30.903,16.482$, and $15.30 \%$, respectively. Furthermore, the best performance of BD-BPANN occurred at 20:30, with a forecasting MAPE of $0.56 \%$; meanwhile, $39.00 \%$ is the worst MAPE of this new forecasting method. The average forecasting MAPE of the price calculated by BD-BPANN is $13.25 \%$, which is less than that of the classical method.

Figure 7 shows the forecasting results of b-BPANN and $\mathrm{BD}-\mathrm{BPANN}$ and the actual electricity price. It is clear that BD-BPANN is superior to BPANN because the green points (forecasted values of BD-BPANN) are closer to the blue curve (actual price) than the red rectangles.
Next, the consequence of using CPSO-BD-BPANN is described as follows. In Table 3, units of demand and price are $\mathrm{Hmk} / \mathrm{h}$ and $\$ / \mathrm{Mwh}$, respectively. The forecasting MAPE of the demand obtained by CPSO-BPANN ranges from $0.12 \%$ at $15: 00$ to $4.55 \%$ at $1: 00$, and the CPSO-BPANN's average forecasting MAPE of the demand is $1.23 \%$, which is less than $1.38 \%$, the result of the b-BPANN. At 22:30, the best DV forecasted value, whose MAPE is $0.009 \%$, appears. The worst MAPE of DV reaches $41.94 \%$ at 8:30, with the average value of the MAPE of DV calculated by CPSO-BPANN of $10.79 \%$, which is still less than the $11.24 \%$ obtained using bBPANN only. In the end, the final model, CPSO-BD-BPANN, is used to forecast the electricity prices of June 9 in 2008. Additionally, to enable a comparison between CPSO-BPANN and CPSO-BD-BPANN, the results of CPSO-BPANN, that is, to calculate the forecasting values of price by only using CPSO-BPANN, are also presented in Table 2. $0.23 \%$ is the best MAPE of CPSO-BPANN at 8:00, and $42.64 \%$ is the worst 

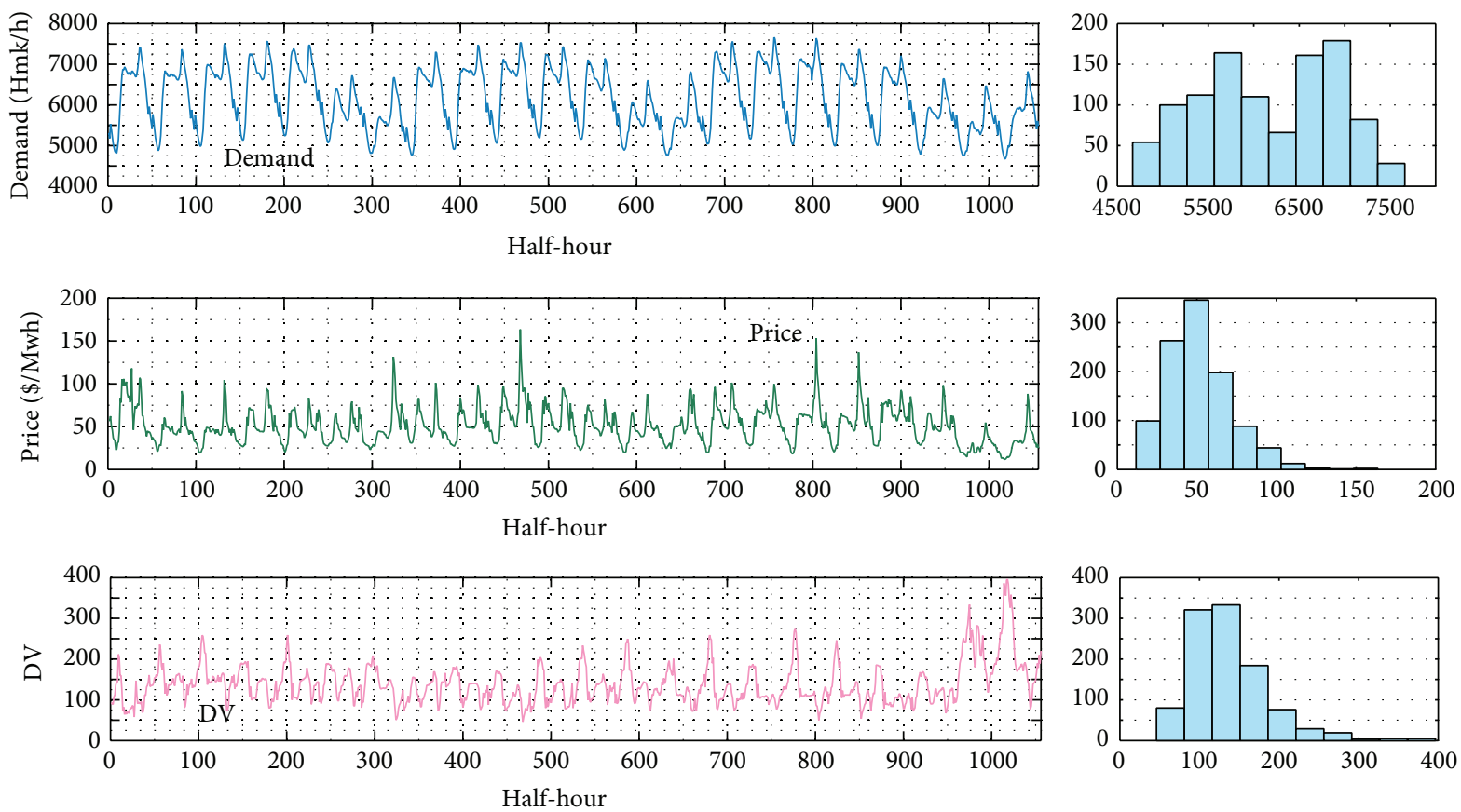

FIGURE 6: Schematic diagram of the electricity price demand and DV.

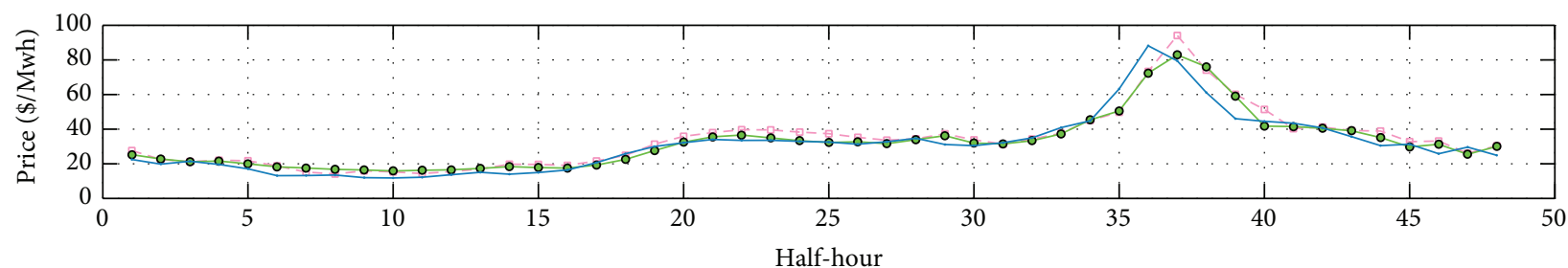

- b-BPANN

$\because$ BD-BPANN

- Actual price

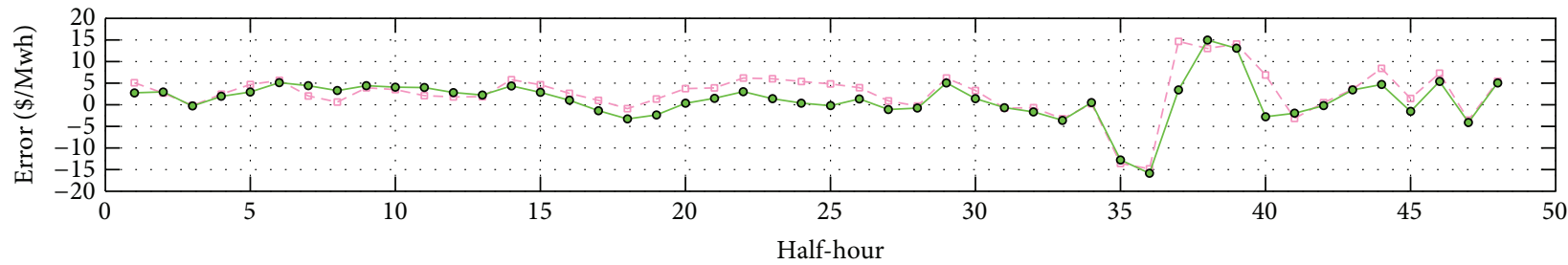

$-\because-\mathrm{b}-\mathrm{BPANN}$

- BD-BPANN
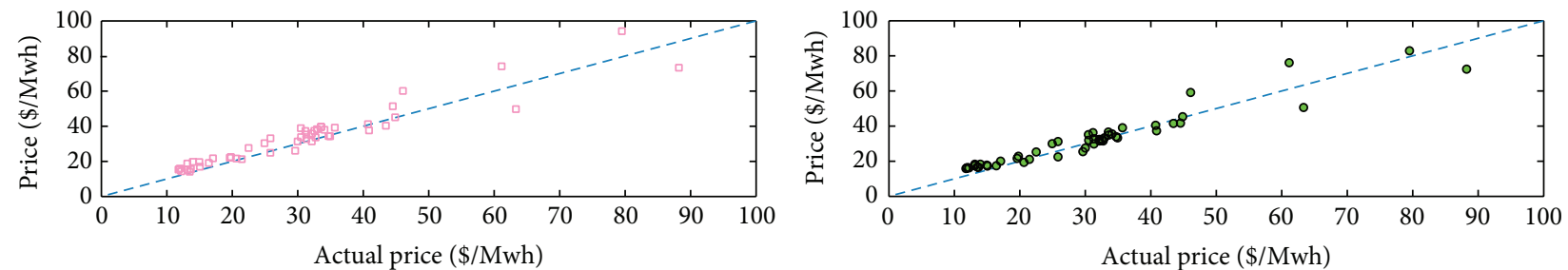

- - Actual

b-BPANN

- - Actual

- BD-BPANN

FIgURE 7: BPANN and BD-BPANN methods for case 1. 


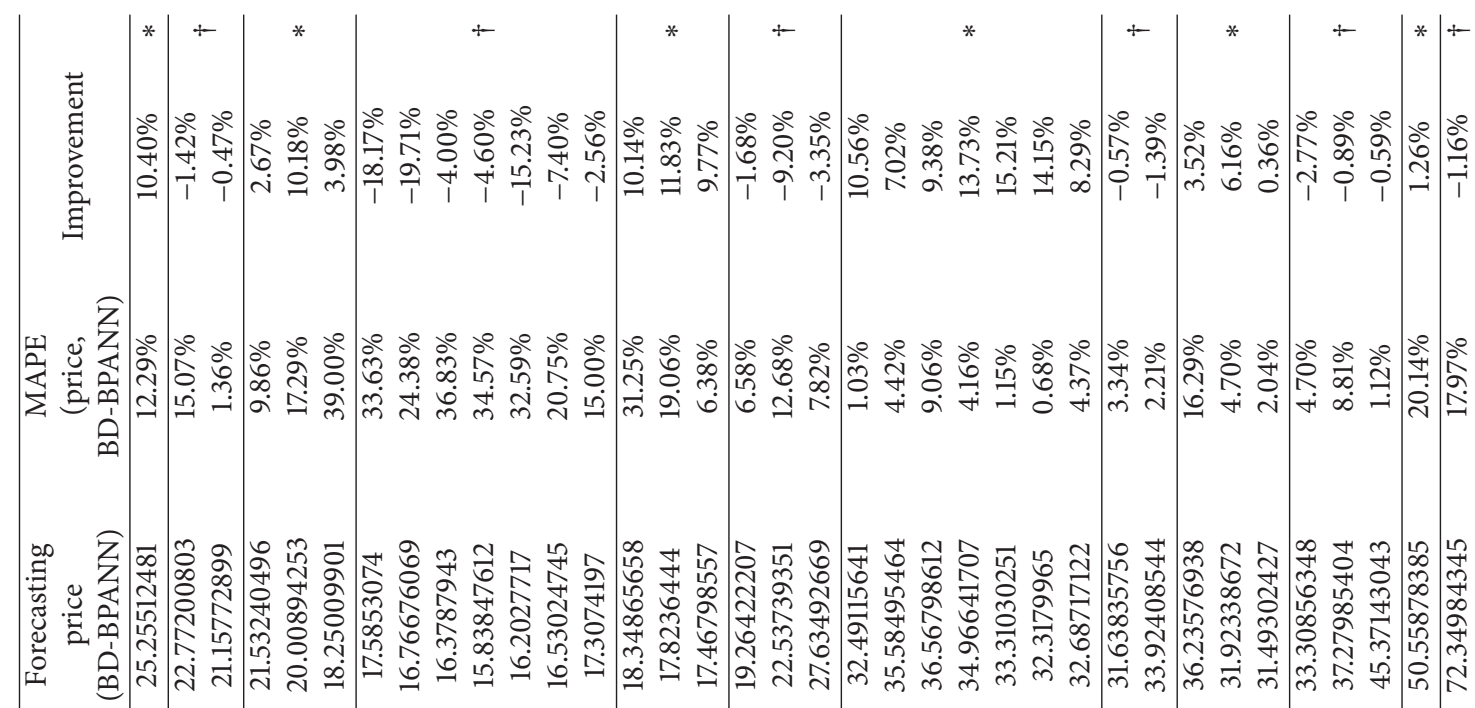

س

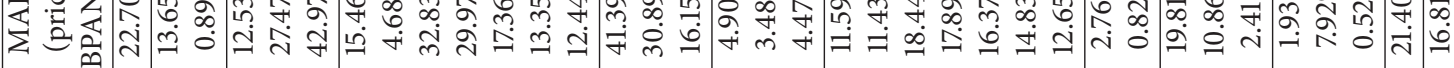

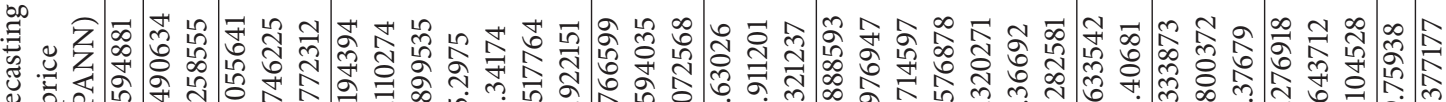

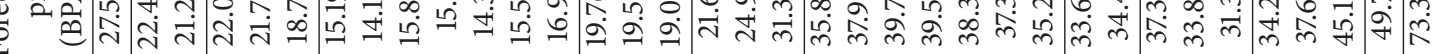

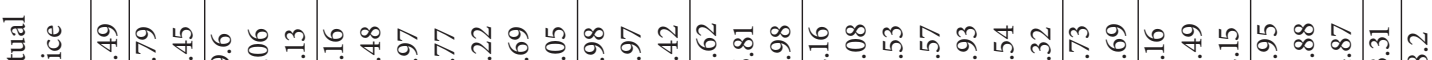

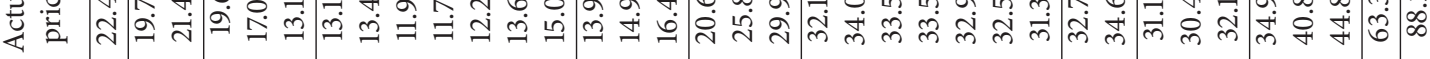

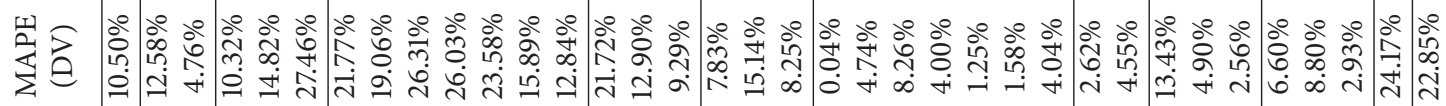

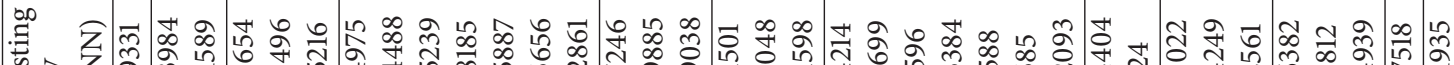

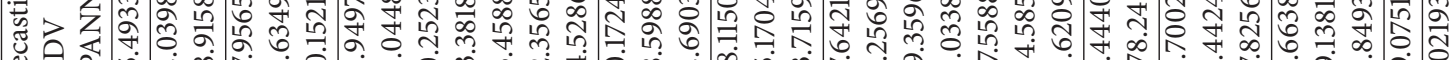
雚

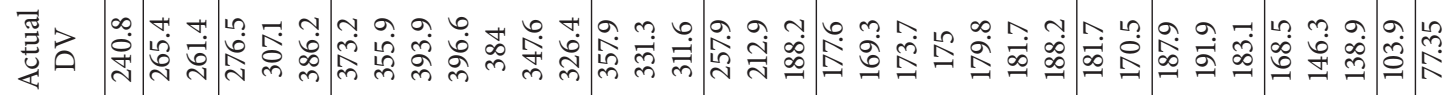

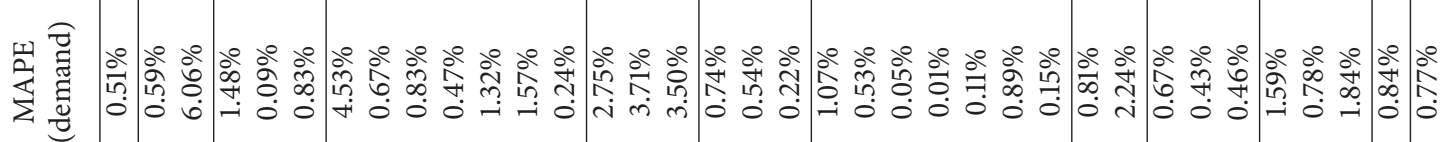

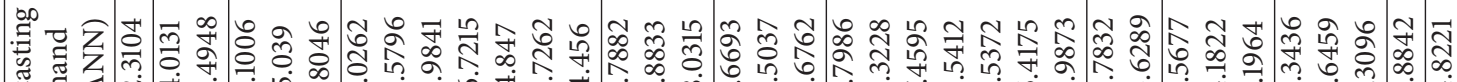

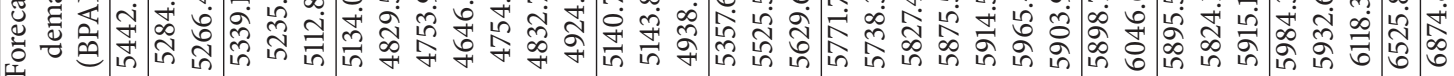

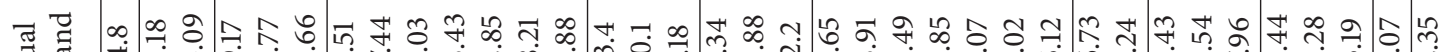

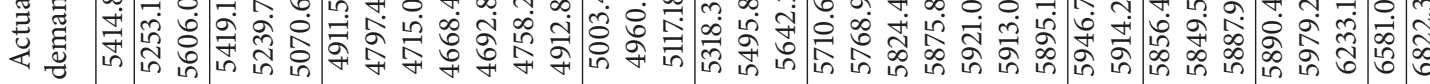
咅 


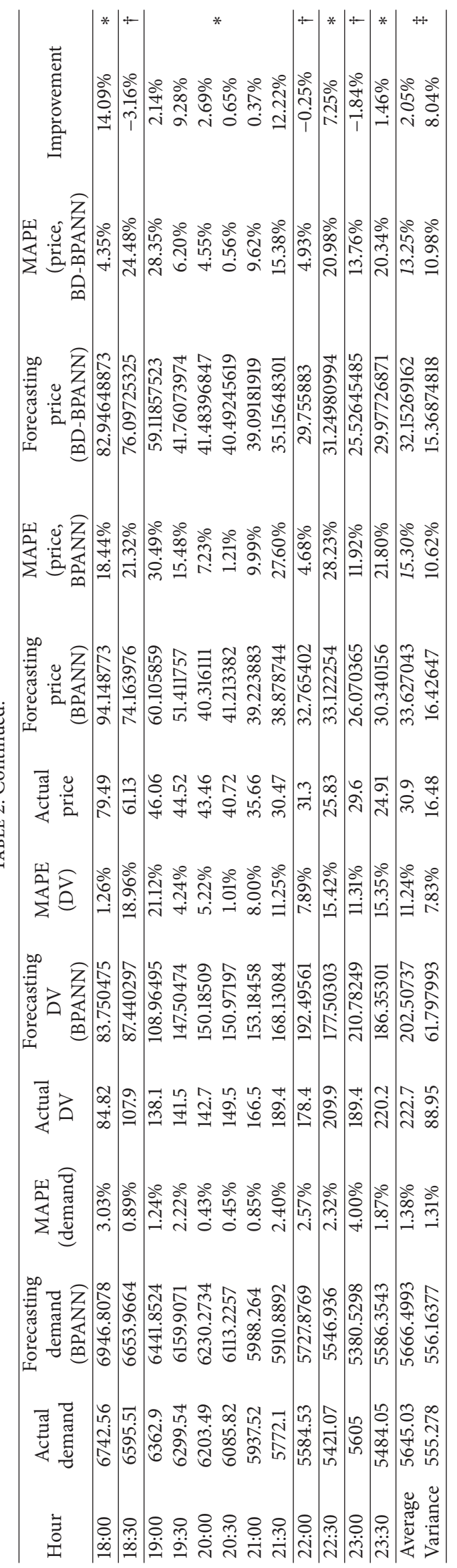


MAPE of the CPSO-BPANN used to forecast electricity price, which occurred at 2:30. In addition, the average MAPE CPSO-BPANN is $14.18 \%$, which is better than that of the bBPANN of $15.30 \%$. In the end, the final model in this paper is used to forecast the electricity price of June 9 in 2008. At 10:00 on this day, the new model yields the best forecasting MAPE, which is only $0.36 \%$. On the contrary, at $2: 30$, the worst performance of CPSO-BD-BPANN, with a MAPE of $43.07 \%$, arises. The average MAPE obtained by CPSO-BDBPANN of this day is $12.31 \%$, which is less than that calculated by CPSO-BPANN. However, there are 28 time spots, that is, the symbol "*" in Table 3, where the effectiveness of CPSOBD-BPANN is better than that of CPSO-BPANN. Figure 8 graphically shows the results presented in Table 3.

Overall, it is not difficult to determine that CPSOBD-BPANN has the best effectiveness among the models of b-BPANN, BD-BPANN, CPSO-BPANN, and CPSO-BDBPANN. Further, the fact that BD-BPANN outperforms CPSO-BPANN, whose calculating time is much longer than that of BD-BPANN, explains why BD-BPANN is more efficient than CPSO-BPANN, which is an optimal artificial neural network under the same conditions, including calculating capacity and language of debugging.

5.3. Study of Case 2. The primary objective of this part is testing the effectiveness of models in spring, rather than explaining the details of the methods, as was performed in case 1. This section will present the effectiveness of each method, as measured by MAPE. Tables 4 and 5 present a comparison between the b-BPANN method and the CPSO-BDBPANN and that between CPSO-BPANN and BD-BPANN. It is obvious that CPSO-BD-BPANN performs better than $\mathrm{b}$ BPANN in all of the spring week except March 11. In March 10, March 13, March 14, and March 16, BD-BPANN is more efficient than CPSO-BPANN, and the overall effectiveness of BD-BPANN is still better than that of CPSO-BPANN in this randomly selected spring week. The results indicate that this sample transformation, named bivariate division, of the input of b-BPANN is more effective than a complex process using an artificial intelligent algorithm, CPSO in this paper, to obtain the best initial parameters of the network.

Specifically, on March 10, CPSO-BPANN exhibited its best MAPE, $0.18 \%$, at 5:30 and obtains its worst one, $103.16 \%$, at $17: 00.1 .16 \%$ is the minimal MAPE of BD-BPANN at 9:00; in contrast, at 14:30, BD-BPANN exhibits its maximum MAPE, $56.10 \%$, of this day. The best MAPE of another model, CPSOBD-BPANN, is $0.0002 \%$ at $8: 30$ and the worst MAPE for this model reaches $46.52 \%$ at $14: 30$ on this spring day. On the next day of this week, March 11, CPSO-BPANN exhibits its best forecasting performance, with a MAPE of $0.02 \%$ at 12:30, and the method exhibited a maximum MAPE, $45.55 \%$, at 17:00. Meanwhile, the smallest MAPE of BDBPANN is $0.09 \%$ at $22: 00$, and its maximum one is $42.89 \%$ at $7: 00.0 .17 \%$ is the best forecasting error by using CPSOBD-BPANN, which exhibited its worst MAPE of $38.52 \%$ at 7:00. On March 12, the MAPE of CPSO-BPANN ranges from $0.04 \%$ at $9: 00$ to $35.03 \%$ at 7:00; that of BD-BPANN reaches its smallest MAPE value, $0.02 \%$, at $11: 30$ and its maximum one, $43.40 \%$, at 17:00; and CPSO-BD-BPANN exhibited its best forecasting effectiveness, with a MAPE of $0.2 \%$, at 19:30, and its worst one, $34.21 \%$, at 17:00. On the fourth day of this spring week, b-BPANN optimized by the CPSO algorithm obtains its minimal MAPE, whose value is $0.08 \%$ at $3: 30$ and its maximum one, $74.99 \%$, at 19:30; the forecasting MAPE of BD-BPANN changes from its smallest value $0.34 \%$ at $17: 00$ to its largest one $62.31 \%$ at $15: 00$ of this day; the final proposed approach, CPSO-BD-BPANN, exhibited its best forecasting effectiveness at 4:00, with a MAPE of $0.23 \%$, and its worst effectiveness at 15:00, with a MAPE of $56.86 \%$. On March 14, CPSO-BPANN exhibited its minimum MAPE of this day of $1.59 \%$, at $21: 30$, and its largest MAPE of 178.85\%, at 19:30; simultaneously, the MAPE of BD-BPANN and CPSO-BD-BPANN ranges from 3.08\% at $5: 30$ and $0.73 \%$, respectively, at $22: 00$ up to $71.38 \%$ and $71.74 \%$ for BD-BPANN and CPSO-BD-BPANN, respectively, at $15: 00$. For the next day, March $15,0.0003 \%$ is the minimum MAPE forecasted by CPSO-BPANN at 3:00, and $52.19 \%$ at $21: 30$ is the maximum one; BD-BPANN exhibited its best forecasting performance with a MAPE of $0.36 \%$ at $6: 00$ and its worst one with a MAPE of $43.13 \%$ at $21: 30$; using CPSOBD-BPANN, its minimum MAPE, $0.34 \%$, and its maximum one, $41.14 \%$, appear at 22:30 and 21:30, respectively. On the last day of this week, the minimum MAPE of CPSO-BPANN is $0.18 \%$ at $4: 00$ and the largest one of this method is $62.99 \%$ at 14:30; simultaneously, the MAPE of both of the new models, BD-BPANN and CPSO-BD-BPANN, changes from $0.06 \%$ at $4: 30$ and $13: 00$, respectively, to values of $67.56 \%$ and $62.48 \%$ for BD-BPANN and CPSO-BD-BPANN, respectively, at 14:30. The results indicate that BD-BPANN and CPSOBD-BPANN both exhibit better forecasting effectiveness than does CPSO-BPANN, especially in the aspect of stability, considering that both BD-BPANN and CPSO-BD-BPANN do not exhibit an extreme MAPE beyond 100\%. Figures 9 and 10 show the results of these 4 forecasting methods through the comparison with the actual price and errors of the forecasting values. Thus, the CPSO-BD-BPANN and BD-BPANN methods outperform CPSO-BPANN and the original b-BPANN.

5.4. Results Analysis and Models Evaluation. In this section, the results of the proposed models of cases 3-5 are presented in Tables 6, 7, and 8. Next, each season's average MAPE of CPSO-BD-BPANN, BD-BPANN, CPSO-BPANN, and bBPANN is presented in Table 9. Simultaneously, the forecasting values of these approaches are also illustrated in pictures. Finally, each of the models will be evaluated through three aspects: forecasting accuracy, calculating time, and stability.

5.4.1. Results Analysis. Table 6 collects the forecasting values calculated by CPSO-BD-BPANN and BD-BPANN. Each column refers to result of each method on different days of summer. For June 9, the lowest MAPE is $0.36 \%$ at 10:00 when using BD-BPANN, which also exhibited the highest MAPE, $43.70 \%$, at 2:30 on this day. The forecasting MAPE of BDBPANN on June 10 ranges from $0.02 \%$ at 3:00 to $44.62 \%$ at 8:30. On June 11, the MAPE values of BD-BPANN ranged 


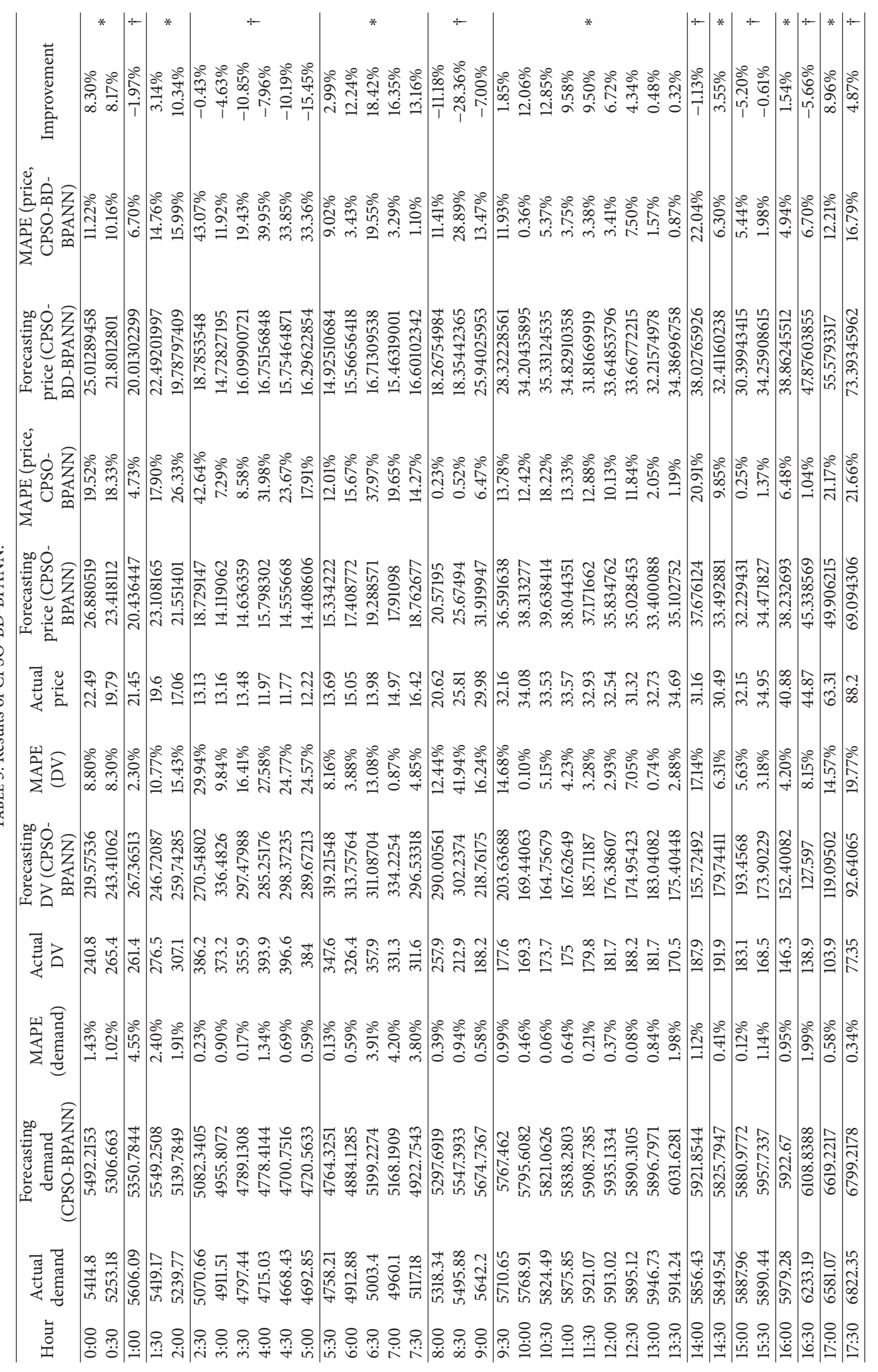




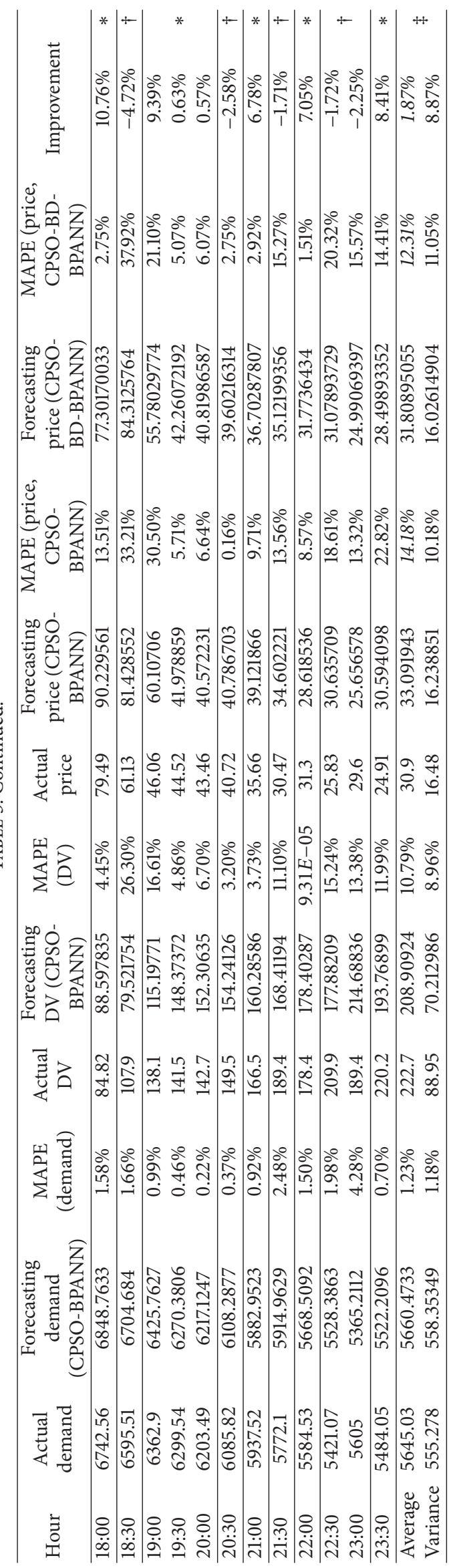




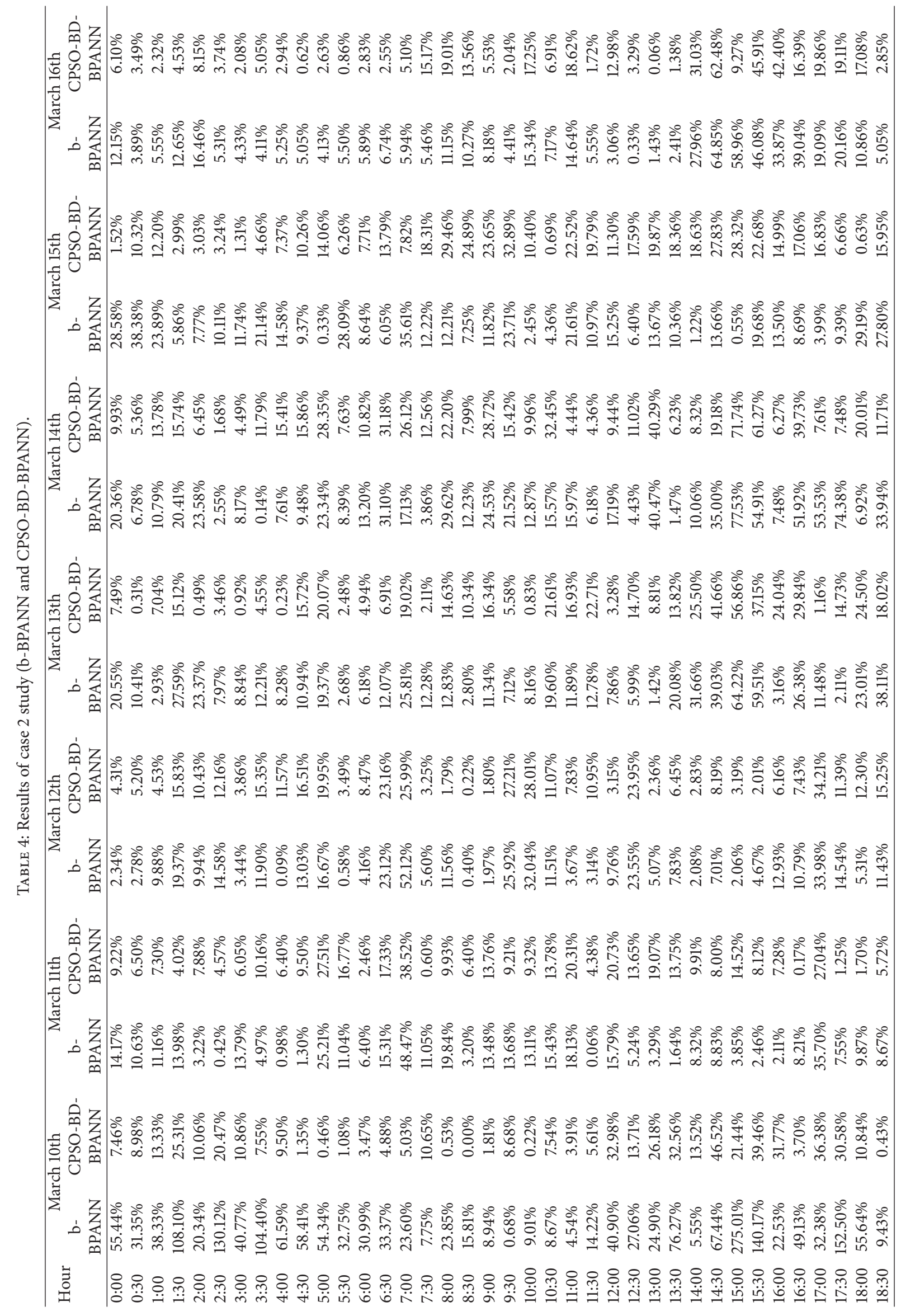




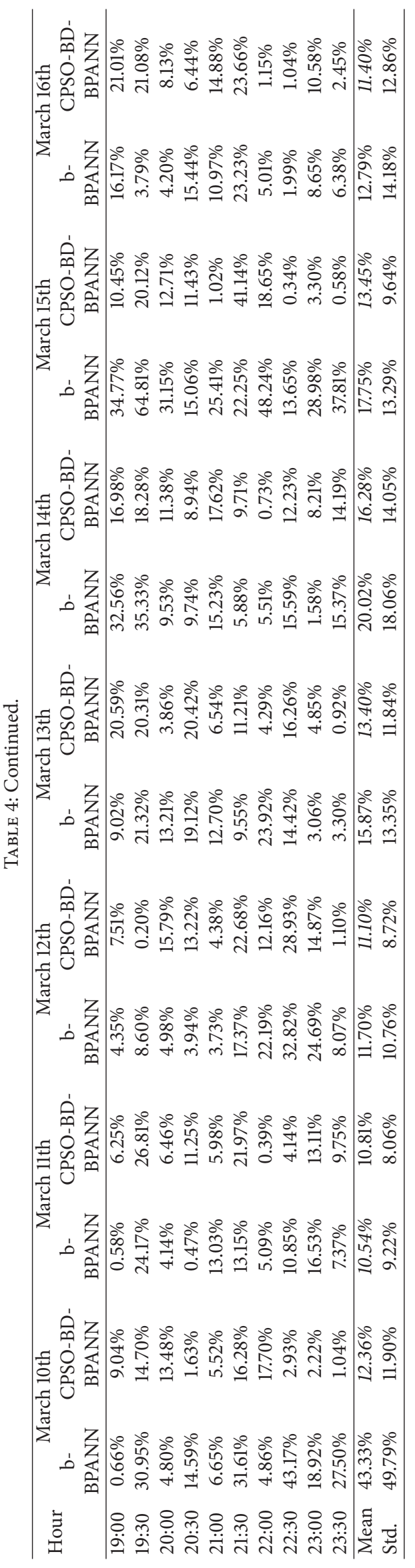




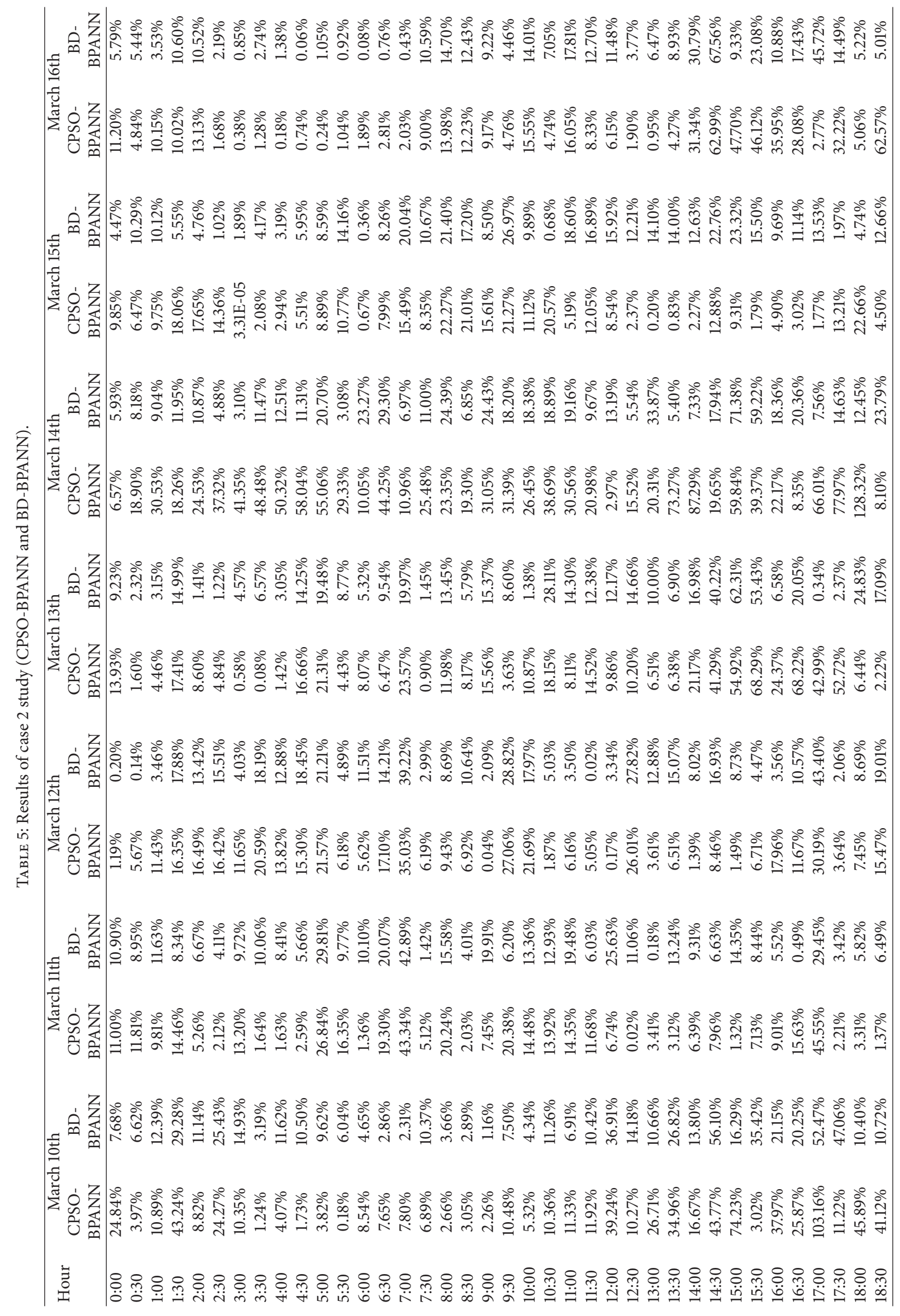




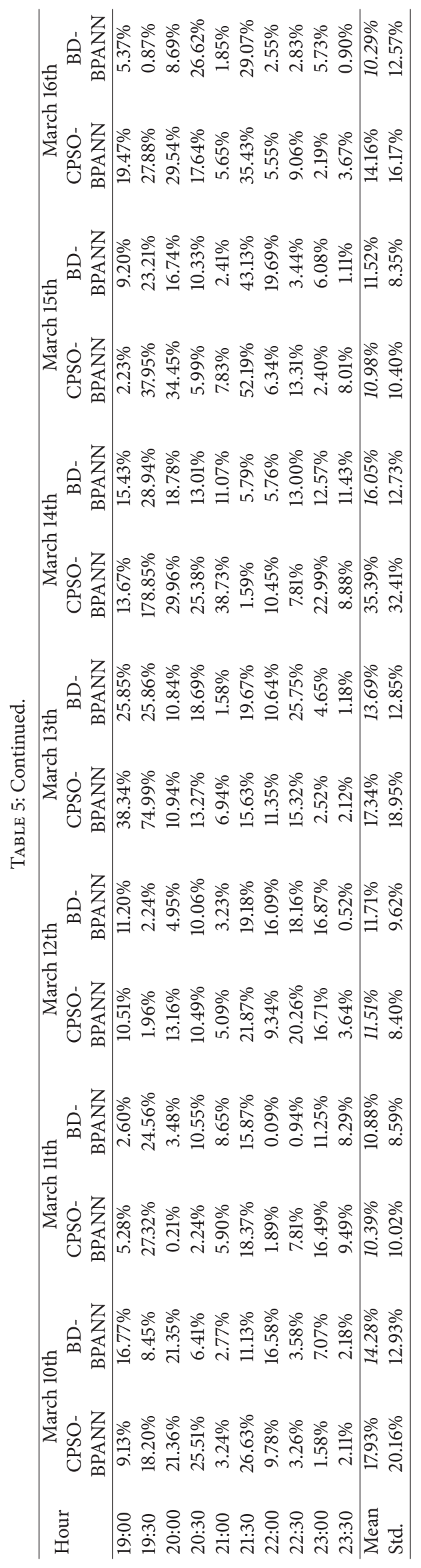



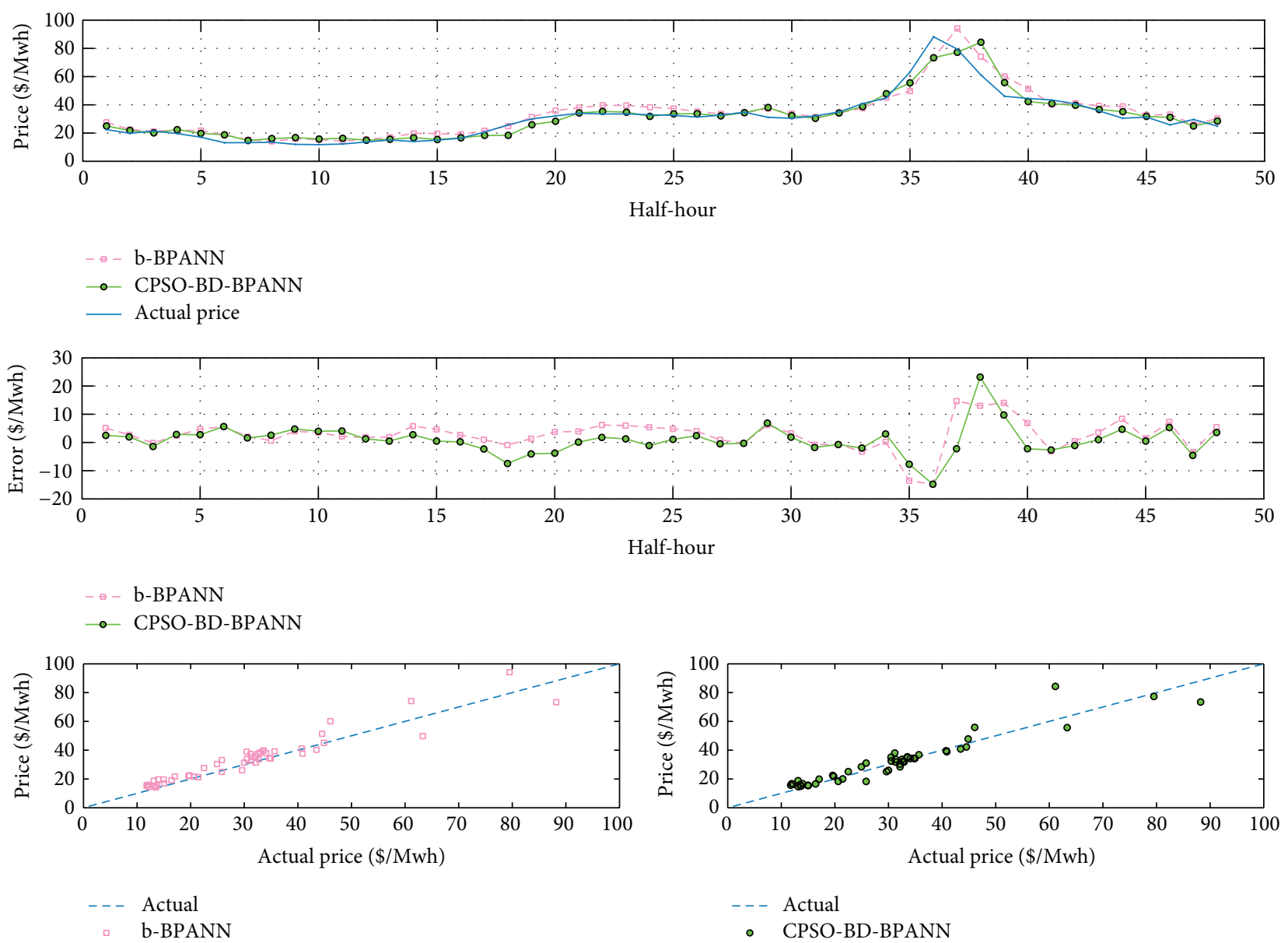

FIGURE 8: BPANN and CPSO-BD-BPANN methods for case 1.

from $0.04 \%$ at $15: 30$ to $41.50 \%$ at $1: 30$. On June 12 of this summer week, CPSO-BD-BPANN has the best performance, whose MAPE is $0.04 \%$ at $16: 00$, while BD-BPANN has the worst one, whose MAPE is $35.42 \%$ at 6:00. The MAPE of the fifth day in this week, June 13 , changes from $0.07 \%$ for CPSOBD-BPANN at $15: 30$ to $32.36 \%$ for BD-BPANN at 17:00. On June 14, the lowest forecasting MAPE of $0.02 \%$ for BDBPANN occurred at 14:00 and CPSO-BD-BPANN exhibited the worst forecasting effectiveness with a MAPE of $31.51 \%$ at 00:00. For the last day of this summer week, the MAPE ranges from $0.001 \%$ at $13: 00$ to $49.06 \%$ at $6: 30$, both of which are for BD-BPANN.

Table 7 shows the MAPE computed through using CPSO-BD-BPANN and BD-BPANN to forecast one week of autumn's electricity price. For September 15, the minimum MAPE is $0.14 \%$ at $13: 00$ when using BD-BPANN to forecast price, while CPSO-BD-BPANN exhibited the maximum MAPE of $35.03 \%$ at $11: 30$. The forecasting MAPE of September 16 changes from $0.12 \%$ obtained by CPSOBD-BPANN at 10:00 to $45.48 \%$ obtained by BD-BPANN at 18:00. On September 17, the lowest MAPE value of $0.08 \%$ at $14: 00$ and the highest MAPE of $157.45 \%$ at 3:00 were both for BD-BPANN. On September 18 of this week, CPSOBD-BPANN exhibited the lowest MAPE of $0.19 \%$ at $11: 30$, while its highest MAPE, $29.32 \%$ at 6:30, is also the highest in this day. The MAPE of the fifth day, September 19, in this autumn week ranges from $0.96 \%$ for CPSO-BD-BPANN at $5: 00$ to $27.53 \%$ for BD-BPANN at 22:00. On September 20 , the lowest forecasting MAPE of $0.08 \%$ for CPSO-BDBPANN was observed at 14:30, while the optimized BDBPANN model exhibited the maximum MAPE of $45.91 \%$ at 9:00 of this day. For the last day of this week, the MAPE changes from $0.28 \%$ at $9: 30$ to $43.43 \%$ at $23: 30$, both for BDBPANN.

Table 8 demonstrates the forecasting values calculated by CPSO-BD-BPANN and BD-BPANN. Each column refers to the result of each method on different days of winter. For December 8 , the lowest MAPE is $0.06 \%$ at $0: 00$ when using CPSO-BD-BPANN, while BD-BPANN exhibits the highest MAPE of $35.00 \%$ at 5:00 on this day. The forecasting MAPE of December 9 ranges from $0.006 \%$ at $12: 00$ to $34.43 \%$ at $1: 30$ which are calculated through CPSO-BD-BPANN and BDBPANN, respectively. On December 10, the MAPE of CPSOBD-BPANN was $0.30 \%$ at 0:30 which is the lowest MAPE, while BD-BPANN exhibited the highest MAPE of $25.18 \%$ at 23:00. On December 11 of this winter week, CPSO-BDBPANN has the best performance, whose MAPE is $0.26 \%$ at 10:30, while BD-BPANN exhibited the worst MAPE of 


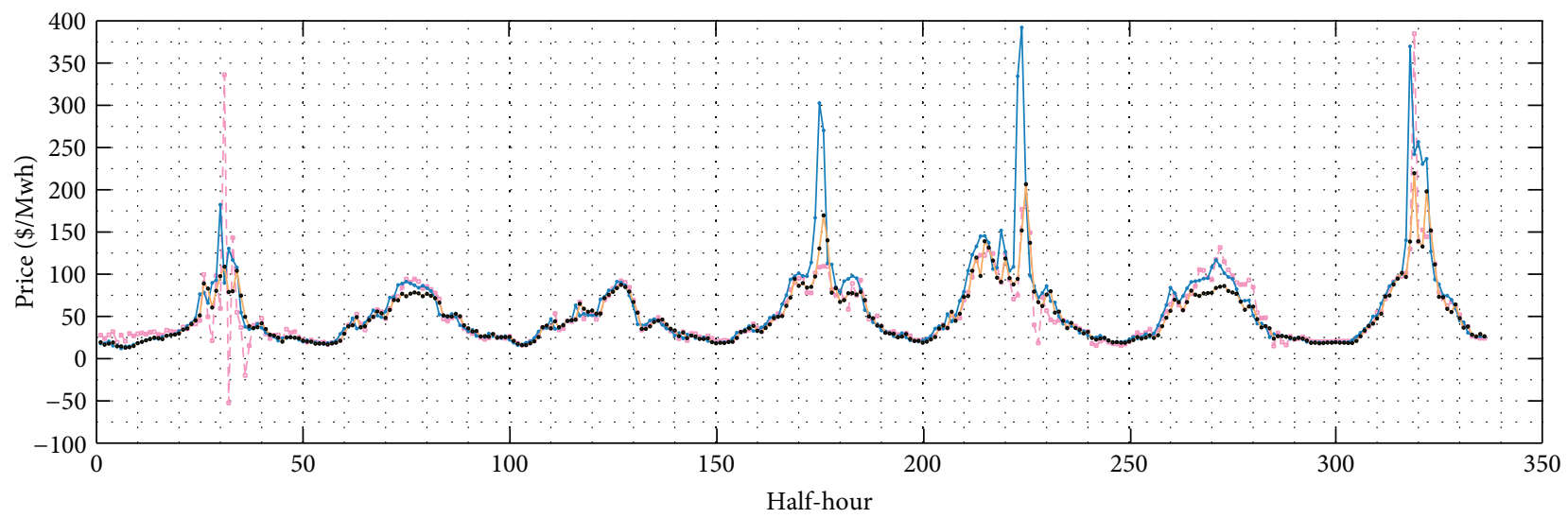

- - b-BPANN

- Actual price

- CPSO-BD-BPANN
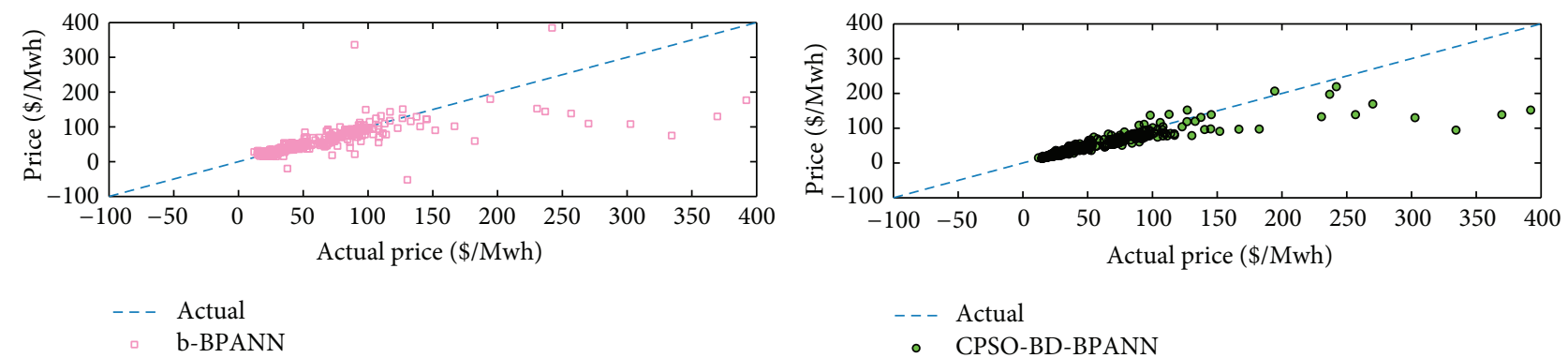

FIGURE 9: BPANN and CPSO-BD-BPANN methods for case 2.

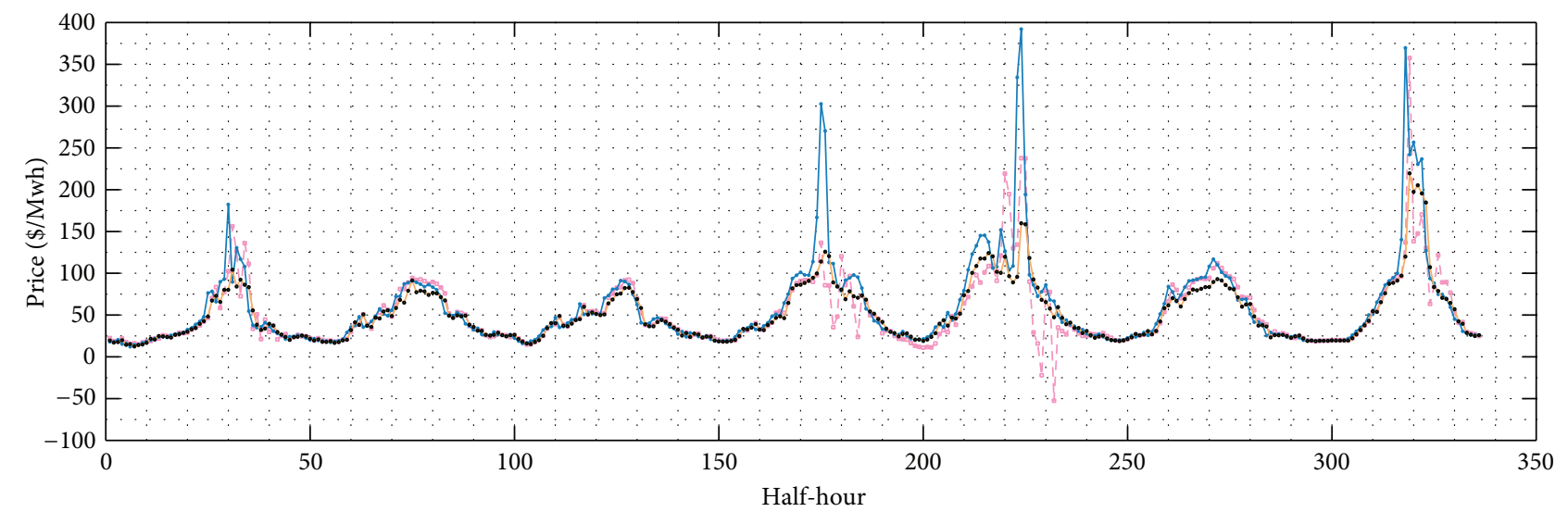

- - CPSO-BPANN

- Actual price

$\therefore$ BD-BPANN
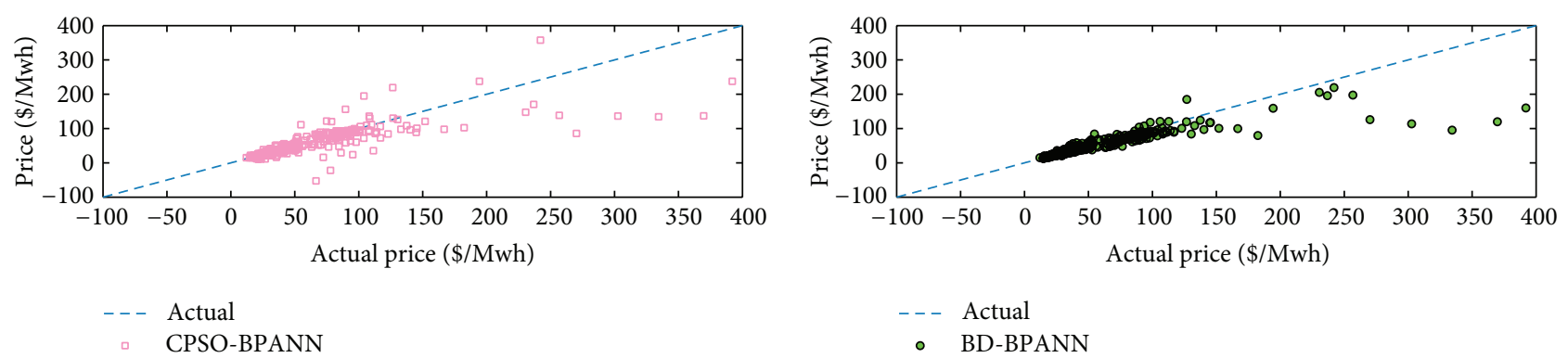

Figure 10: BD-BPANN and CPSO-BPANN methods for case 2. 


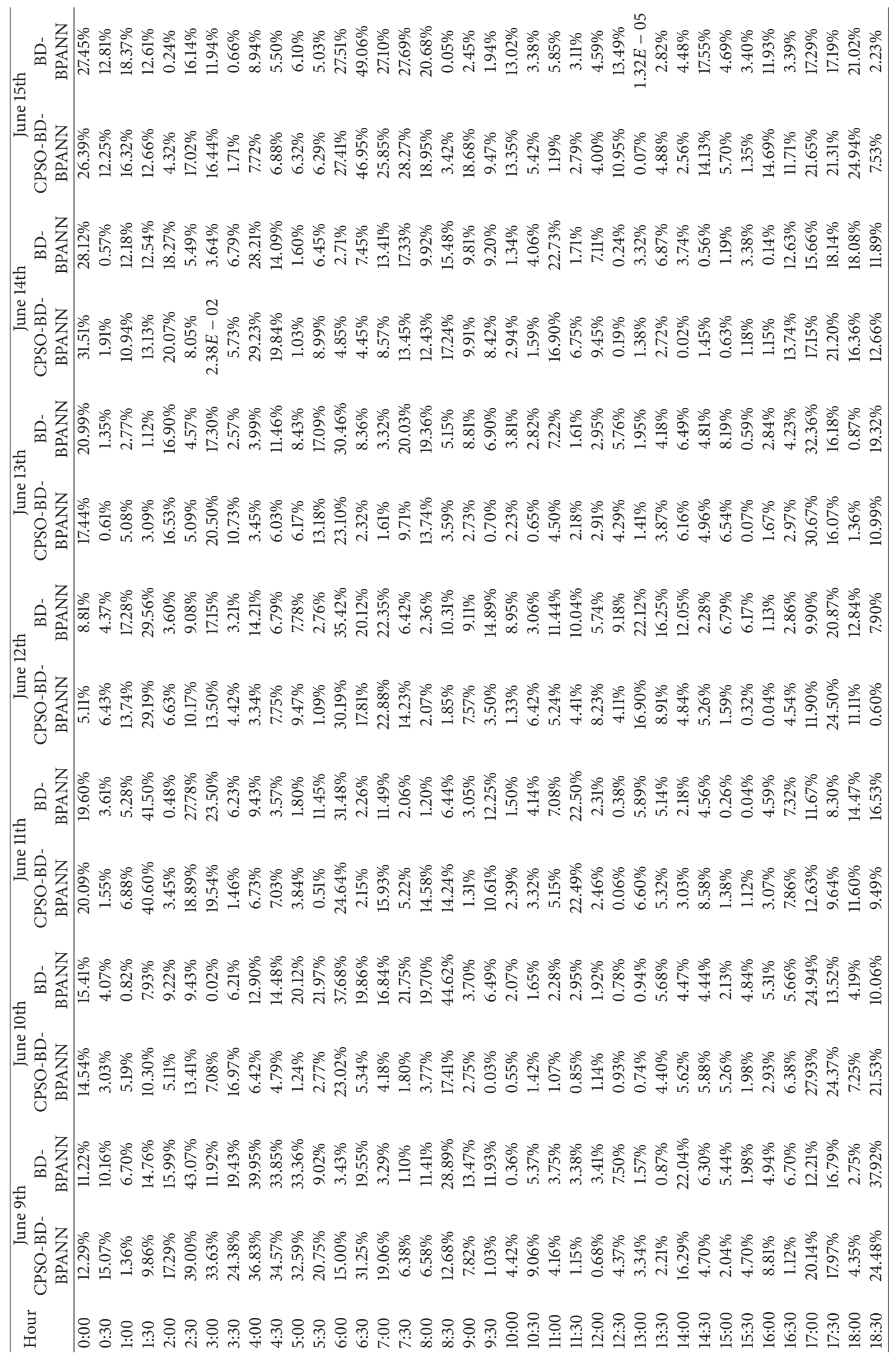




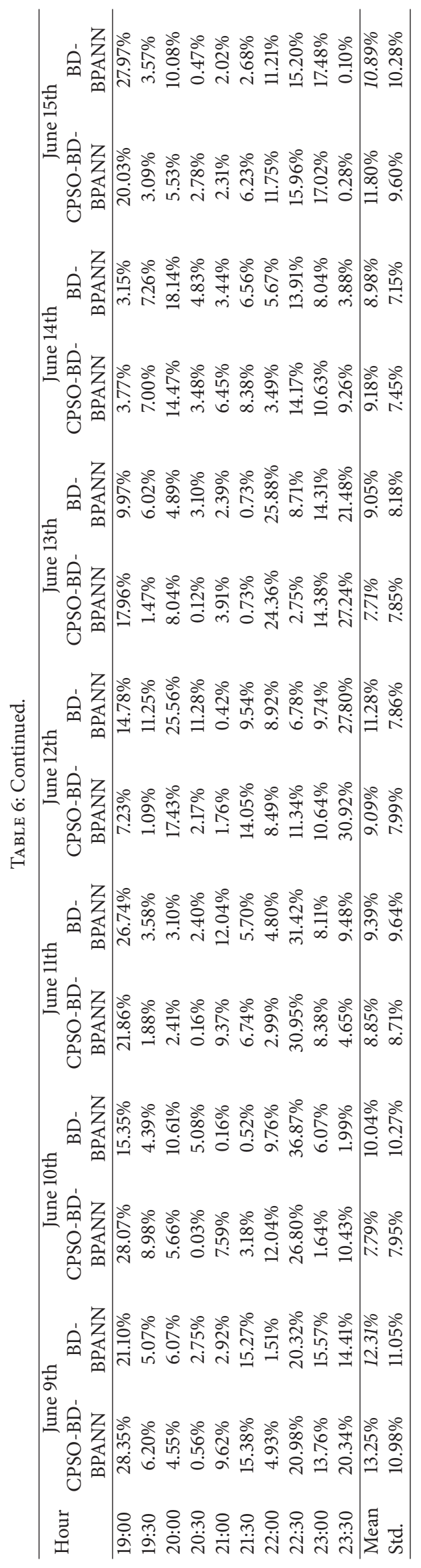




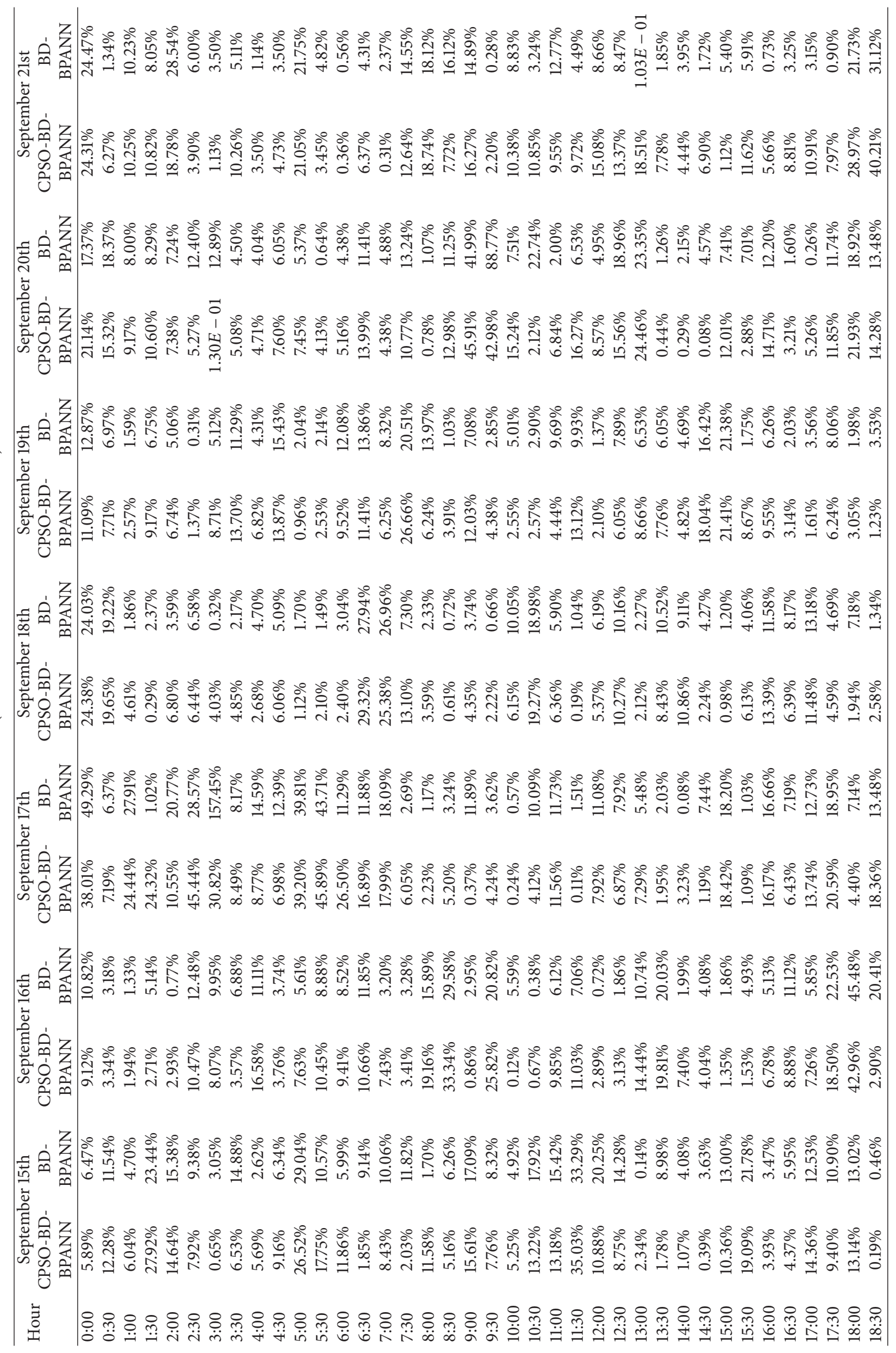




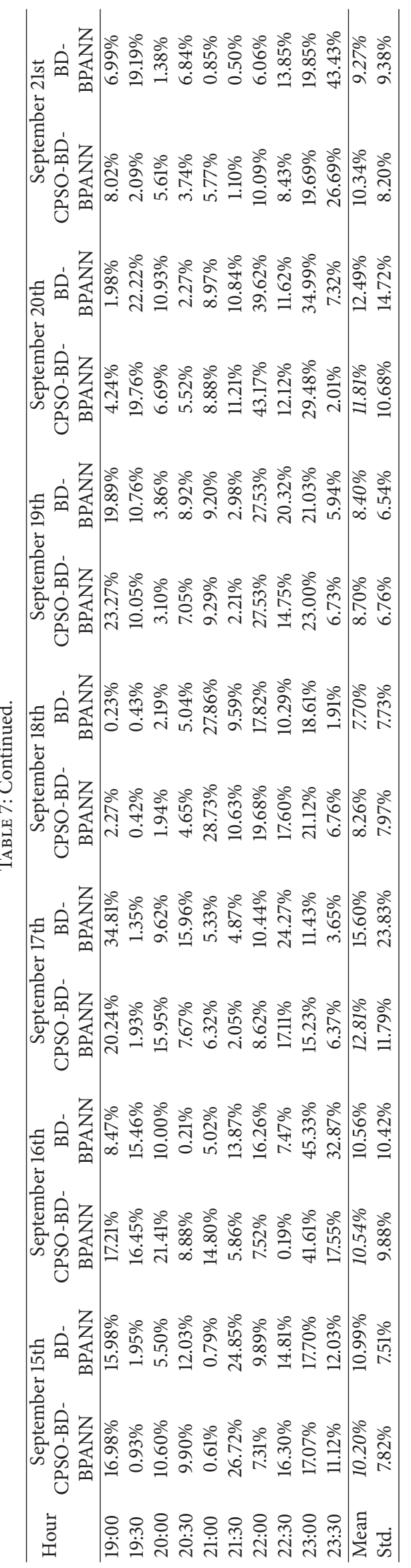




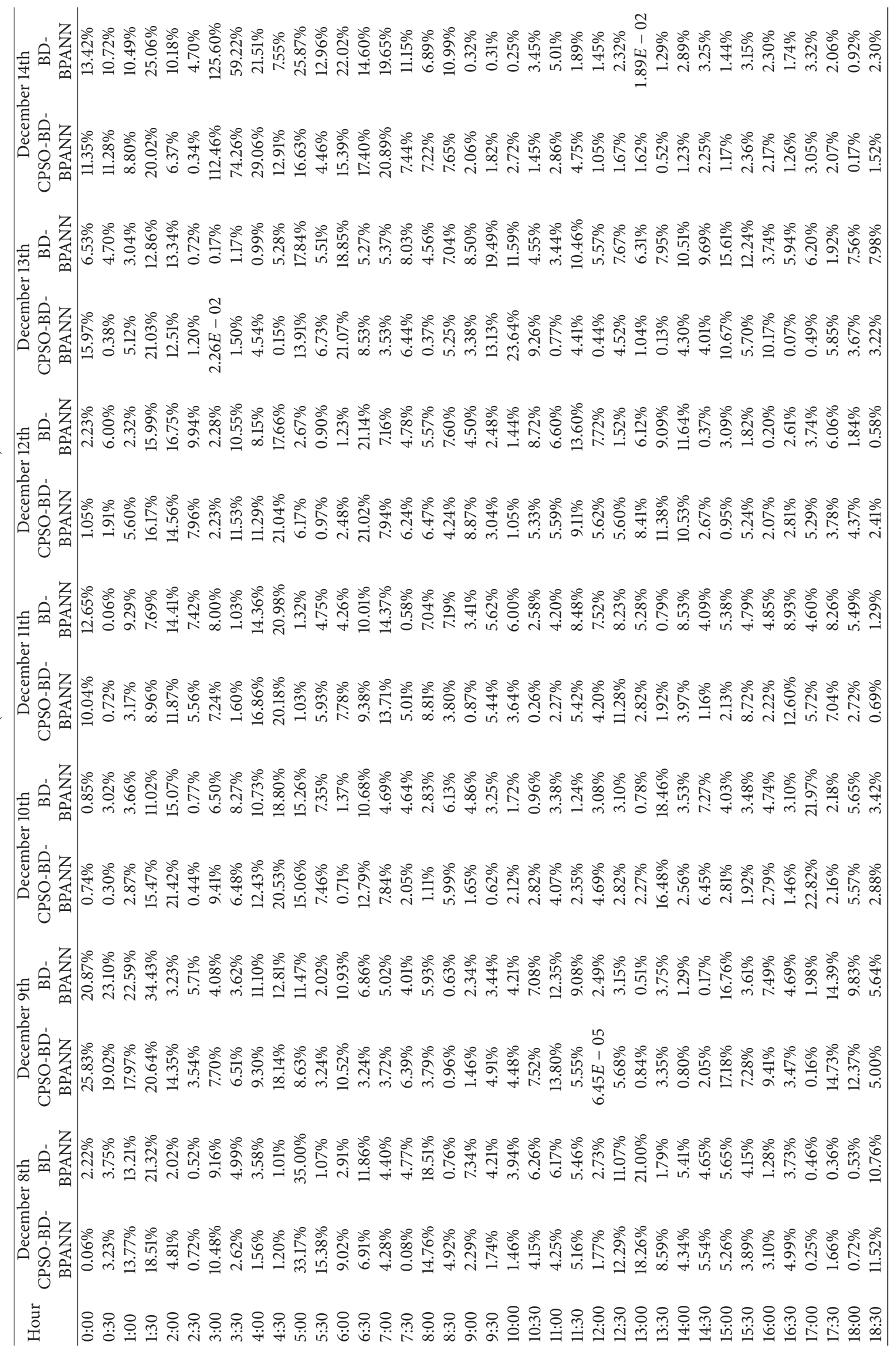




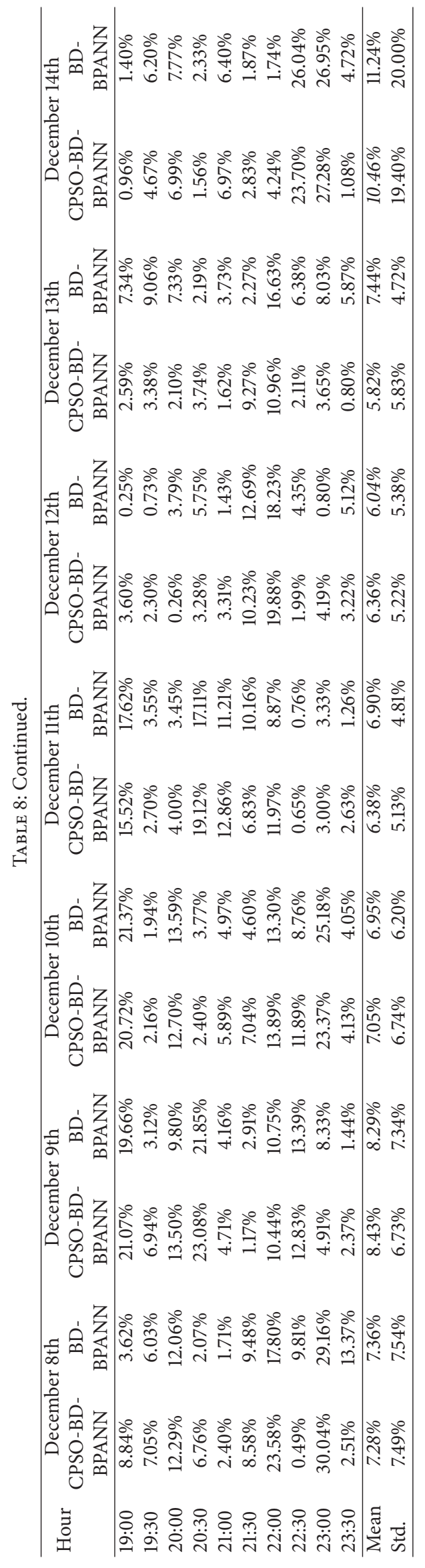


TABLE 9: Models evaluation.

\begin{tabular}{|c|c|c|c|c|c|}
\hline Season & Evaluation items & b-BPANN & CPSO-BPANN & BD-BPANN & CPSO-BD-BPANN \\
\hline \multirow{3}{*}{ Spring } & Accuracy (MAPE) & $18.86 \%$ & $16.81 \%$ & $12.68 \%$ & $12.63 \%$ \\
\hline & Calculating time (seconds) & 3.25 & 88.36 & 6.81 & 162.29 \\
\hline & Index of stability & 6 & 2 & 0 & 0 \\
\hline \multirow{3}{*}{ Summer } & Accuracy (MAPE) & $11.26 \%$ & $11.46 \%$ & $10.28 \%$ & $9.67 \%$ \\
\hline & Calculating time (seconds) & 3.41 & 88.03 & 6.92 & 161.35 \\
\hline & Index of stability & 1 & 0 & 0 & 0 \\
\hline \multirow{3}{*}{ Autumn } & Accuracy (MAPE) & $11.27 \%$ & $11.37 \%$ & $10.72 \%$ & $10.38 \%$ \\
\hline & Calculating time (seconds) & 3.45 & 88.98 & 6.99 & 168.89 \\
\hline & Index of stability & 1 & 1 & 0 & 0 \\
\hline \multirow{3}{*}{ Winter } & Accuracy (MAPE) & $8.61 \%$ & $7.99 \%$ & $7.74 \%$ & $7.40 \%$ \\
\hline & Calculating time (seconds) & 3.25 & 84.36 & 6.49 & 159.01 \\
\hline & Index of stability & 4 & 2 & 1 & 1 \\
\hline \multirow{3}{*}{ Average } & Accuracy (MAPE) & $12.50 \%$ & $11.91 \%$ & $10.36 \%$ & $10.02 \%$ \\
\hline & Calculating time (seconds) & 3.34 & 87.4325 & 6.8025 & 162.885 \\
\hline & Index of stability & 3 & 1.25 & 0.25 & 0.25 \\
\hline
\end{tabular}

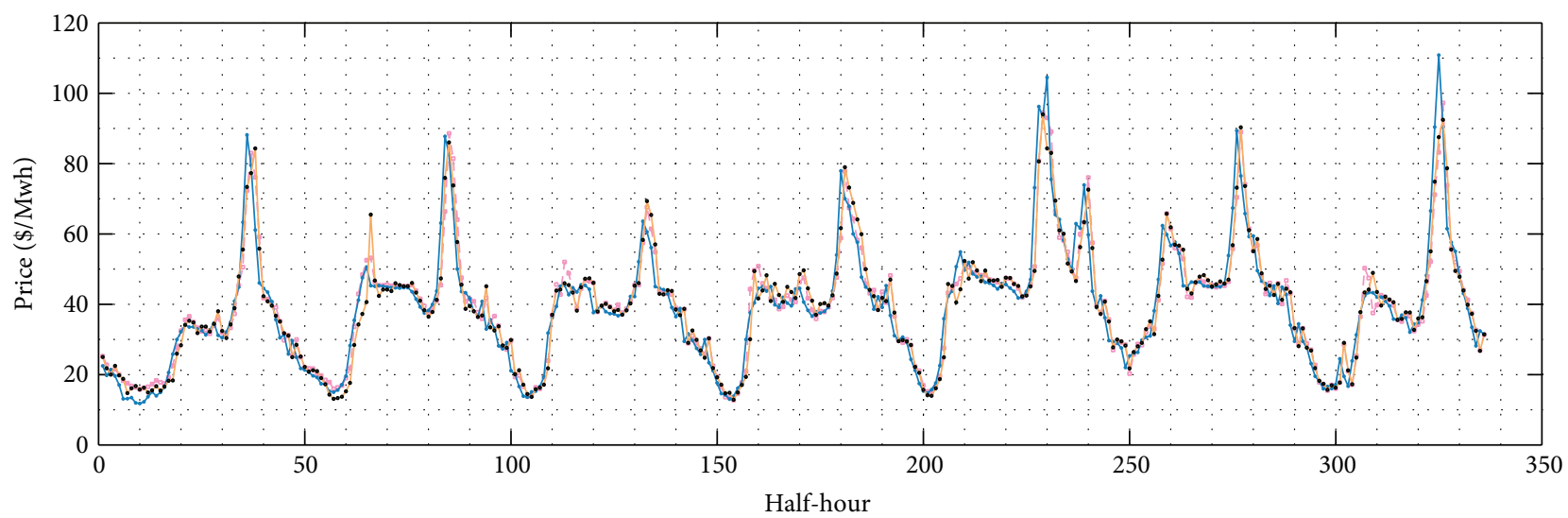

- BD-BPANN

- Actual price

- CPSO-BD-BPANN
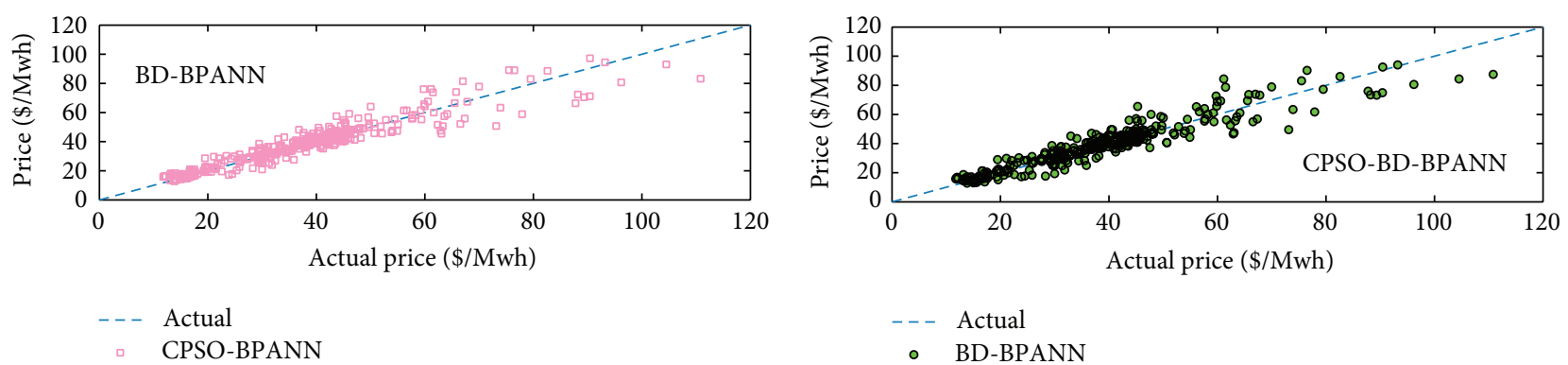

Figure 11: BD-BPANN and CPSO-BD-BPANN methods for case 3.

$20.98 \%$ at $4: 30$. The MAPE of the fifth day in this week, December 12 , changes from $0.20 \%$ at $16: 00$ to $21.14 \%$ at $6: 30$, with both computed by BD-BPANN. On December 13, the lowest forecasting MAPE is $0.07 \%$, calculated by the CPSOBD-BPANN at 16:30, and CPSO-BD-BPANN exhibited the worst forecasting effectiveness, with a MAPE of $23.64 \%$ at
10:00 on this day. For the last day of this winter week, the MAPE ranges from $0.17 \%$ for CPSO-BD-BPANN at $18: 00$ to $125.60 \%$ for BD-BPANN at 3:00.

Overall, in summer, the average forecasting MAPE of the CPSO-BD-BPANN method is $9.67 \%$, which is less than that of BD-BPANN; the forecasting MPAE of BD-BPANN 

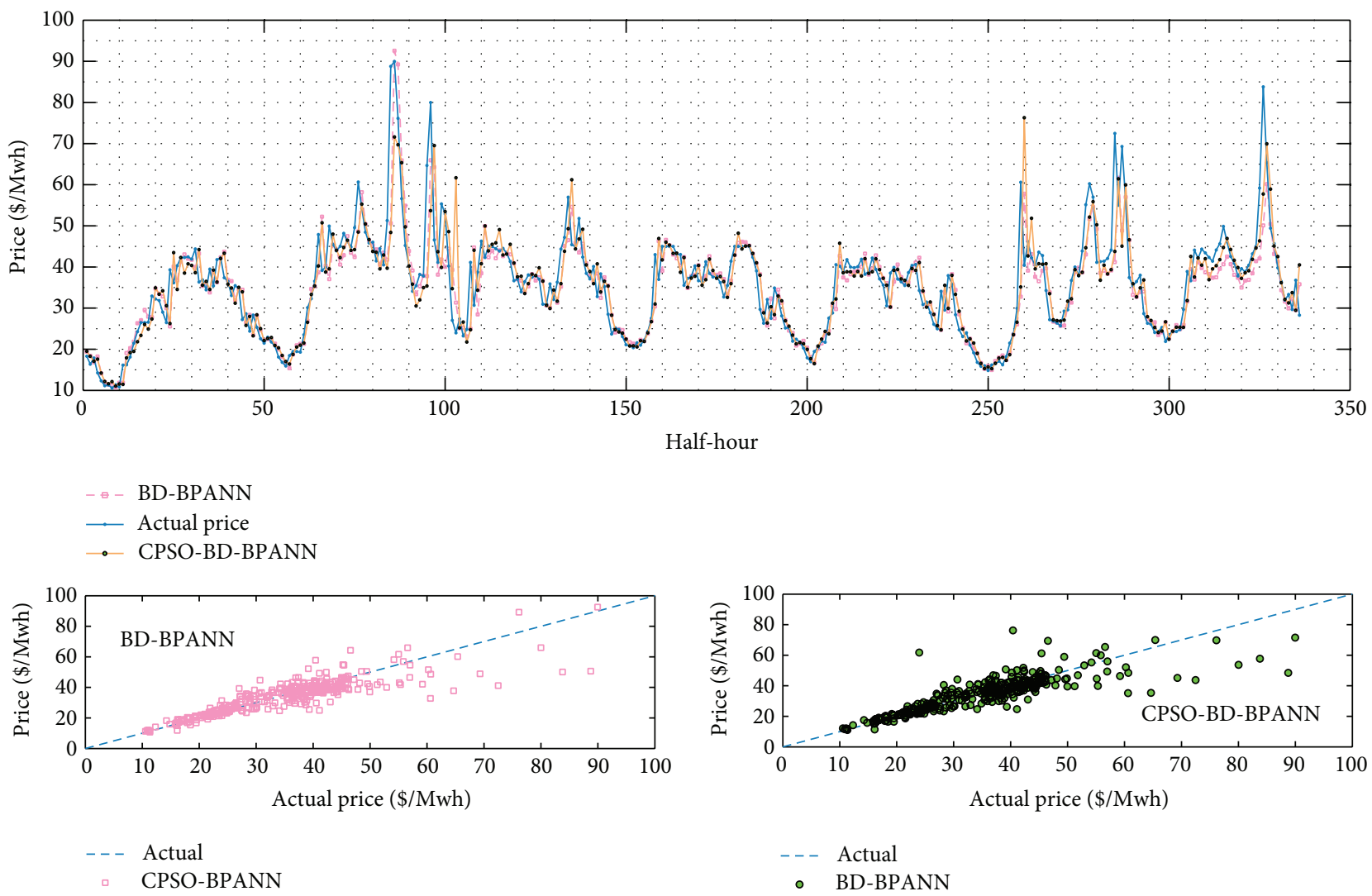

FIGURE 12: BD-BPANN and CPSO-BD-BPANN methods for case 4.

in autumn, $10.72 \%$, is larger than that of CPSO-BD-BPANN, $10.38 \%$. Similarly, in winter, CPSO-BD-BPANN forecasts the electricity price more accurately than the original method, BD-BPANN; the MAPE of CPSO-BD-BPANN of $7.40 \%$ is less than the MAPE of BD-BPANN of 7.74\%. Overall, CPSOBD-BPANN outperforms BD-BPANN 12 times: June 10, June 11, June 12, and June 13 in the summer, September 15, September 16, September 17, and September 20 in autumn, and December 8, December 11, December 13, and December 14 in winter. In contrast, BD-BPANN outperforms CPSOBD-BPANN 9 times. However, as summarized above, CPSOBD-BPANN is a better forecasting model than BD-BPANN. Figures 11, 12, and 13 show the results of the new approaches, the actual values, and the forecasting errors.

5.4.2. Models Evaluation. In the above portion of 5.4, the results of both proposed forecasting models of cases 3, 4, and 5 were presented in tables and figures. These results indicate that CPSO-BD-BPANN outperforms BD-BPANN in the aspect of accuracy. Furthermore, to test the forecasting effectiveness of CPSO-BD-BPANN and BD-BPANN, this paper compares both of them with b-BPANN and CPSOBPANN in the aspects of forecasting accuracy, calculation time, and stability. In this study, the forecasting accuracy is measured by MAPE, and the index of stability of the models is the number of points of the forecasting MAPE that exceed $100 \%$; that is, a model can be regarded as a relatively stable model when the index of stability is relatively low. The unit of calculation time is one second. Table 9 presents the results of the evaluation of the models of b-BPANN, CPSO-BPANN, BD-BPANN, and CPSO-BD-BPANN.

From Table 9, it is obvious that CPSO-BD-BPANN is the best forecasting model when considering accuracy. In the aspect of calculation time, b-BPANN and BD-BPANN significantly outperform the other models. Finally, the last row of this table indicates that the proposed models are better than $\mathrm{b}-\mathrm{BPANN}$ and CPSO-BPANN regarding forecasting stability. As a result, to forecast the electricity price efficiently, either BD-BPANN or CPSO-BD-BPANN should be selected as the forecasting model. Specifically, when accuracy is the only concern, CPSO-BD-BPANN should be used to forecast the electricity price. However, when the cost of calculation is the only concern, BD-BPANN is the best model because its accuracy is higher than that of BPANN, the forecasting accuracy of which must be less than that of b-BPANN (by the definition of b-BPANN).

\section{Our Contribution}

(i) To combine historical price and demand to improve the forecasting of the electricity price, this paper proposed a new variable, known as DV, through a division of one variable by the other, rather than simply regarding them as inputs of a network. In this 


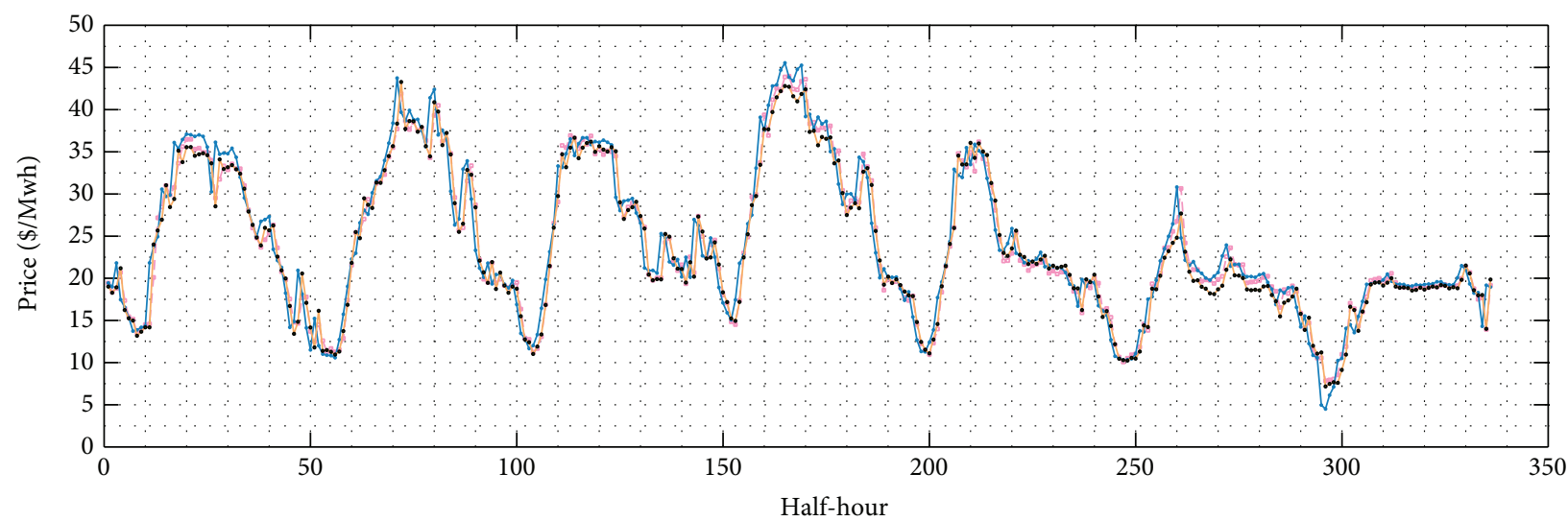

$-\because-\mathrm{BD}-\mathrm{BPANN}$

- Actual price

- CPSO-BD-BPANN

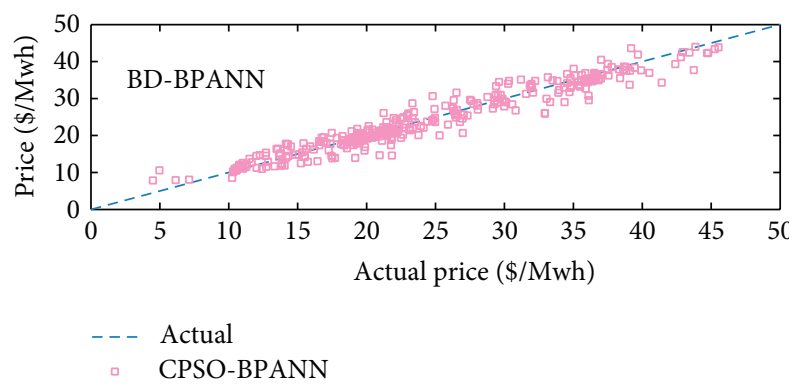

FIGURE 13: BD-BPANN and CPSO-BD-BPANN methods for case 5.

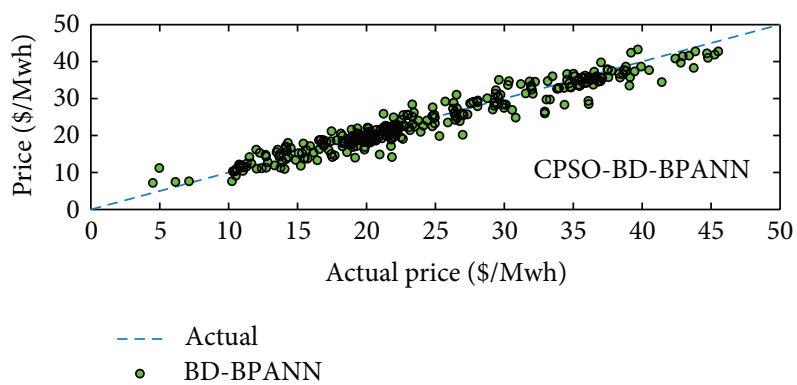

- BD-BPANN approach, multiplication of the forecasted DV and the demand is taken as the final forecasted price, which consequently improves the accuracy of forecasting price than that using the original BPANN.

(ii) The CPSO algorithm, BPANN, and the concept of bivariate division are combined to form the new models: BD-BPANN and CPSO-BD-BPANN.

(iii) This paper evaluated the models through three aspects: forecasting accuracy, calculating time, and stability. The evaluation indicated that CPSO-BDBPANN is a relatively better model when only considering accuracy and that BD-BPANN is a comparatively better model when the cost of calculation should be low, while the accuracy remains high.

\section{Conclusions}

In this paper, the BD-BPANN method is creatively proposed by combining the concept of bivariate division with BPANN to forecast electricity price more accurately and stably than BPANN does. In four seasons of 2008, the average MAPE of $\mathrm{BD}-\mathrm{BPANN}$ is $10.36 \%$, which is less than those of BPANN and CPSO-BPANN. Note that BD-BPANN performs better than BPANN and CPSO-BPANN in each season. Next, the CPSO algorithm was applied to optimize the initial parameters of BD-BPANN to improve the forecasting accuracy. This optimized forecasting model, named CPSO-BDBPANN, forecasted the price with an average MAPE, $10.02 \%$, in the weeks of the four seasons considered. Additionally, the CPSO-BD-BPANN method outperforms the other approaches in forecasting accuracy. Finally, the evaluation of the models of BPANN, BD-BPANN, CPSO-BPANN, and CPSO-BD-BPANN was performed. This evaluation involved tests regarding accuracy, calculation time, and stability. For the aspect of accuracy, CPSO-BD-BPANN is the best model. The BPANN model, which exhibits an average calculation time of 3.34 seconds, has huge advantages compared with the other models regarding the calculation time. The last test, stability, indicated that the proposed methods, both of which have an index of stability of 0.25 , are more stable than the original BPANN and the CPSO-BPANN models, with indices of stability of 3 and 1.5 , respectively. These results illustrate that CPSO-BD-BPANN is a superior model to the others when only concerning accuracy. And BD-BPANN outperforms CPSO-BD-BPANN, CPSO-BPANN, and BPANN when calculation time is the crucial factor.

\section{Highlights}

The highlights of this paper are as follows: skillful transformation of the electricity price and demand into a new variable, known as DV, rather than only regarding both of 
them as inputs of the network; utilization of chaos particle swarm optimization to obtain the relatively improved weights and thresholds of the network, rather than the random ones; proposal of two different forecasting strategies for the user of the model.

\section{Conflict of Interests}

The authors declare that there is no conflict of interests regarding the publication of this paper.

\section{Acknowledgment}

The work was supported by the National Natural Science Foundation of China (Grant no. 71171102).

\section{References}

[1] S. Anbazhagan and N. Kumarappan, "Day-ahead deregulated electricity market price classification using neural network input featured by DCT,' International Journal of Electrical Power and Energy Systems, vol. 37, no. 1, pp. 103-109, 2012.

[2] S. K. Aggarwal, L. M. Saini, and A. Kumar, "Electricity price forecasting in deregulated markets: a review and evaluation," International Journal of Electrical Power and Energy Systems, vol. 31, no. 1, pp. 13-22, 2009.

[3] H.-T. Pao, "Forecasting electricity market pricing using artificial neural networks," Energy Conversion and Management, vol. 48, no. 3, pp. 907-912, 2007.

[4] X. R. Li, C. W. Yu, S. Y. Ren, C. H. Chiu, and K. Meng, "Dayahead electricity price forecasting based on panel cointegration and particle filter," Electric Power Systems Research, vol. 95, pp. 66-76, 2013.

[5] J. Zhang, Z. Tan, and S. Yang, "Day-ahead electricity price forecasting by a new hybrid method," Computers and Industrial Engineering, vol. 63, no. 3, pp. 695-701, 2012.

[6] Z. Tan, J. Zhang, J. Wang, and J. Xu, "Day-ahead electricity price forecasting using wavelet transform combined with ARIMA and GARCH models," Applied Energy, vol. 87, no. 11, pp. 36063610, 2010.

[7] N. Amjady and F. Keynia, "Day-ahead price forecasting of electricity markets by a new feature selection algorithm and cascaded neural network technique," Energy Conversion and Management, vol. 50, no. 12, pp. 2976-2982, 2009.

[8] V. Vahidinasab, S. Jadid, and A. Kazemi, "Day-ahead price forecasting in restructured power systems using artificial neural networks," Electric Power Systems Research, vol. 78, no. 8, pp. 1332-1342, 2008.

[9] A. I. Arciniegas and I. E. Arciniegas Rueda, "Forecasting shortterm power prices in the Ontario Electricity Market (OEM) with a fuzzy logic based inference system," Utilities Policy, vol. 16, no. 1, pp. 39-48, 2008.

[10] S. Chakravarty and P. K. Dash, "Dynamic filter weights neural network model integrated with differential evolution for dayahead price forecasting in energy market," Expert Systems with Applications, vol. 38, no. 9, pp. 10974-10982, 2011.

[11] S. Bordignon, D. W. Bunn, F. Lisi, and F. Nan, "Combining dayahead forecasts for British electricity prices," Energy Economics, vol. 35, pp. 88-103, 2013.
[12] H. Shayeghi and A. Ghasemi, "Day-ahead electricity prices forecasting by a modified CGSA technique and hybrid WT in LSSVM based scheme," Energy Conversion and Management, vol. 74, pp. 482-491, 2013.

[13] S. Anbazhagan and N. Kumarappan, "Day-ahead deregulated electricity market price forecasting using neural network input featured by DCT," Energy Conversion and Management, vol. 78, pp. 711-719, 2014.

[14] D. Niu, D. Liu, and D. D. Wu, "A soft computing system for day-ahead electricity price forecasting," Applied Soft Computing Journal, vol. 10, no. 3, pp. 868-875, 2010.

[15] W.-M. Lin, H.-J. Gow, and M.-T. Tsai, "An enhanced radial basis function network for short-term electricity price forecasting," Applied Energy, vol. 87, no. 10, pp. 3226-3234, 2010.

[16] X. Yan and N. A. Chowdhury, "Mid-term electricity market clearing price forecasting: a hybrid LSSVM and ARMAX approach," International Journal of Electrical Power and Energy Systems, vol. 53, no. 1, pp. 20-26, 2013.

[17] X. Yan and N. A. Chowdhury, "Mid-term electricity market clearing price forecasting: a multiple SVM approach," International Journal of Electrical Power and Energy Systems, vol. 58, pp. 206-214, 2014.

[18] W.-M. Lin, H.-J. Gow, and M.-T. Tsai, "Electricity price forecasting using enhanced probability neural network," Energy Conversion and Management, vol. 51, no. 12, pp. 2707-2714, 2010.

[19] D. Singhal and K. S. Swarup, "Electricity price forecasting using artificial neural networks," International Journal of Electrical Power and Energy Systems, vol. 33, no. 3, pp. 550-555, 2011.

[20] B. D. Rodrigues and M. J. Stevenson, "Takeover prediction using forecast combinations," International Journal of Forecasting, vol. 29, no. 4, pp. 628-641, 2013.

[21] N. An, W. Zhao, J. Wang, D. Shang, and E. Zhao, "Using multi-output feedforward neural network with empirical mode decomposition based signal filtering for electricity demand forecasting," Energy, vol. 49, no. 1, pp. 279-288, 2013.

[22] X. W. Li, S. J. Cho, and S. T. Kim, "Combined use of BP neural network and computational integral imaging reconstruction for optical multiple-image security," Optics Communications, vol. 315, pp. 147-158, 2014.

[23] R. Hecht-Nelson, "Kolmogorov's mapping neural network existence theorem," in Proceedings of 1st IEEE Annual International Conference on Neural Networks, pp. 11-13, IEEE Press, San Diego, Calif, USA, 1987.

[24] Z.-H. Guo, J. Wu, H.-Y. Lu, and J.-Z. Wang, "A case study on a hybrid wind speed forecasting method using BP neural network," Knowledge-Based Systems, vol. 24, no. 7, pp. 10481056, 2011.

[25] J. Wang, Z. Sheng, B. Zhou, and S. Zhou, "Lightning potential forecast over Nanjing with denoised sounding-derived indices based on SSA and CS-BP neural network," Atmospheric Research, vol. 137, pp. 245-256, 2014. 


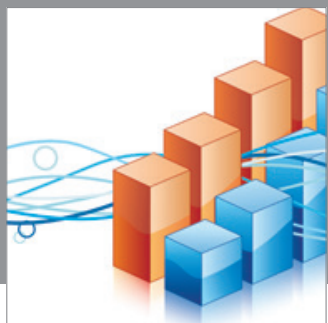

Advances in

Operations Research

mansans

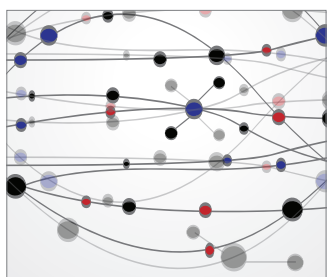

The Scientific World Journal
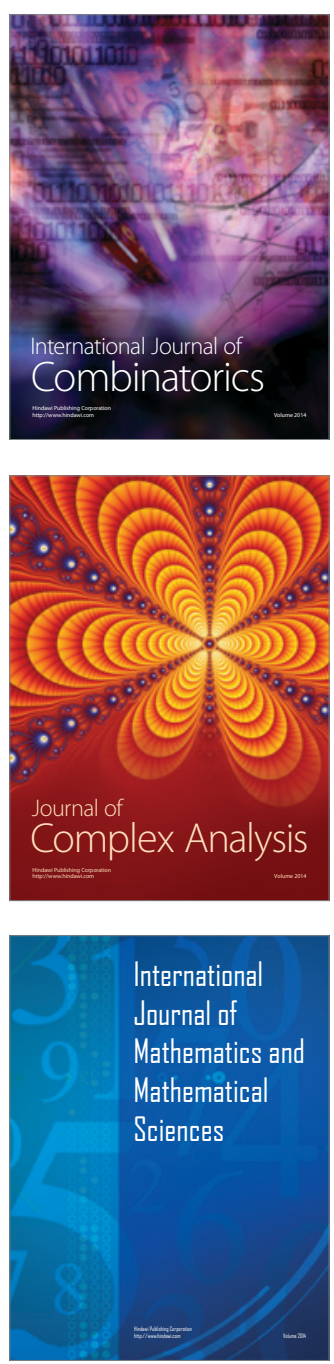
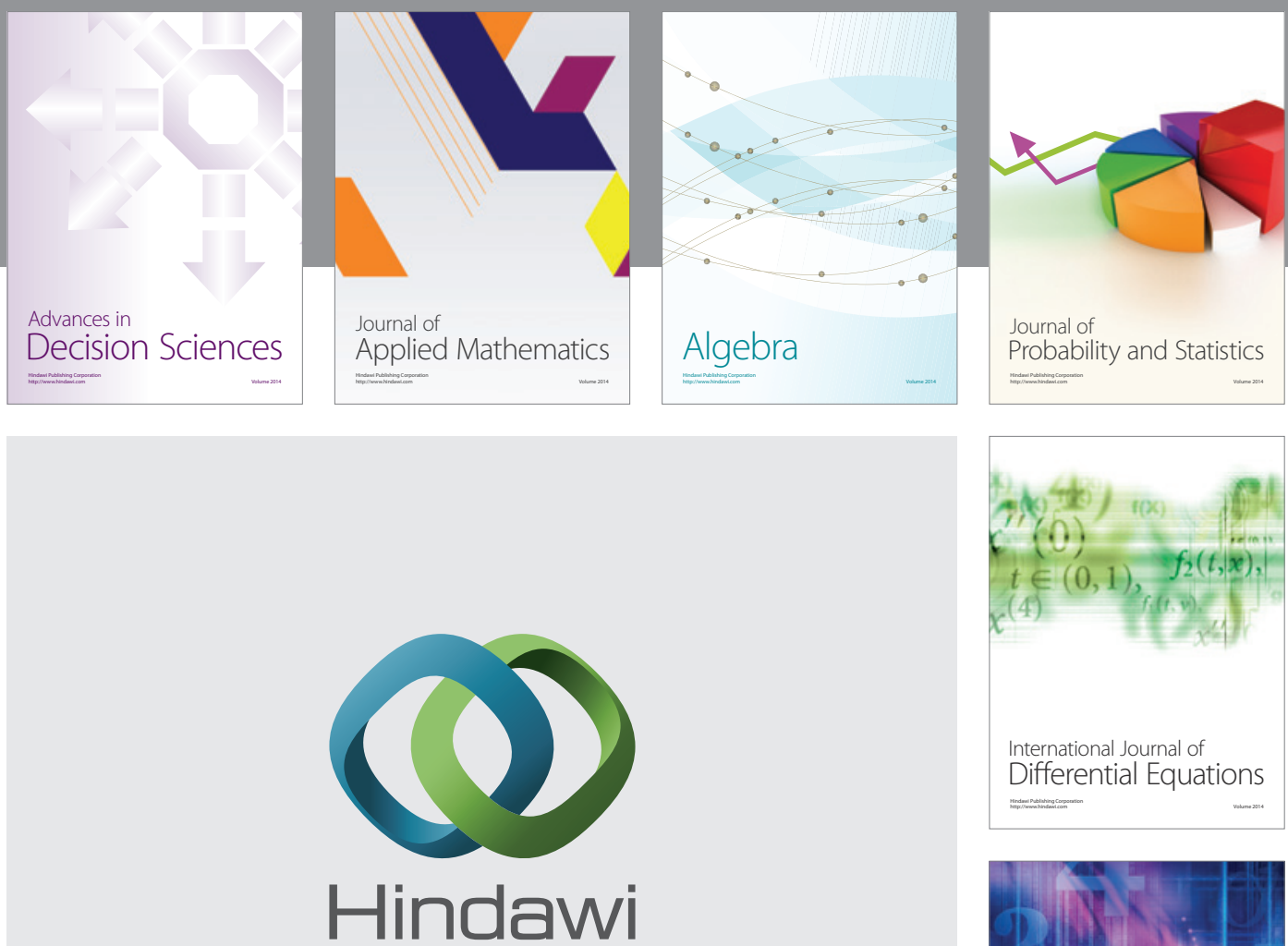

Submit your manuscripts at http://www.hindawi.com
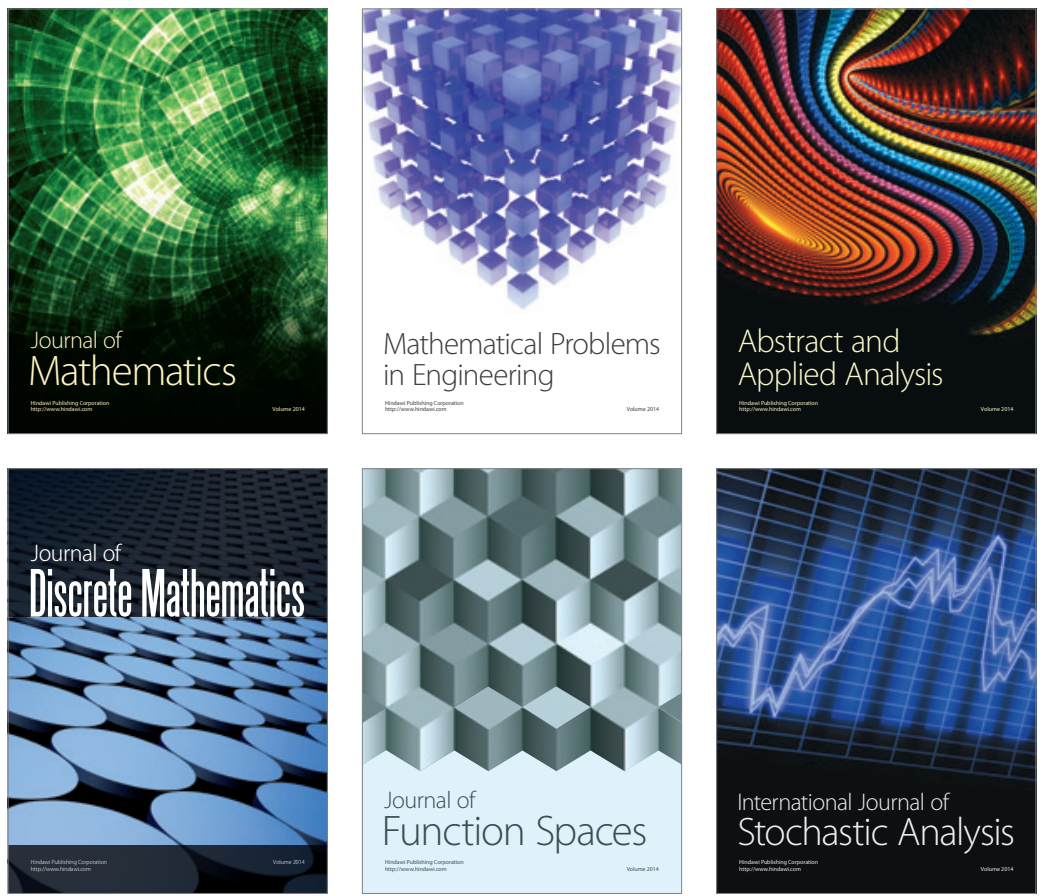

Journal of

Function Spaces

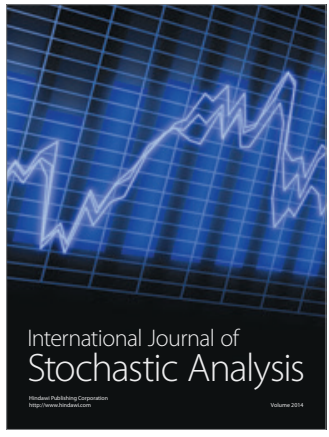

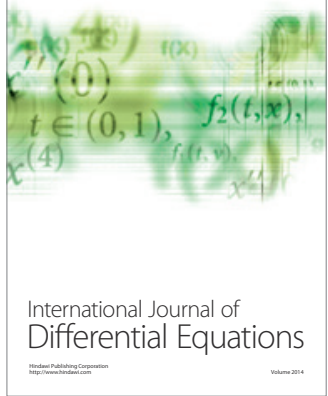
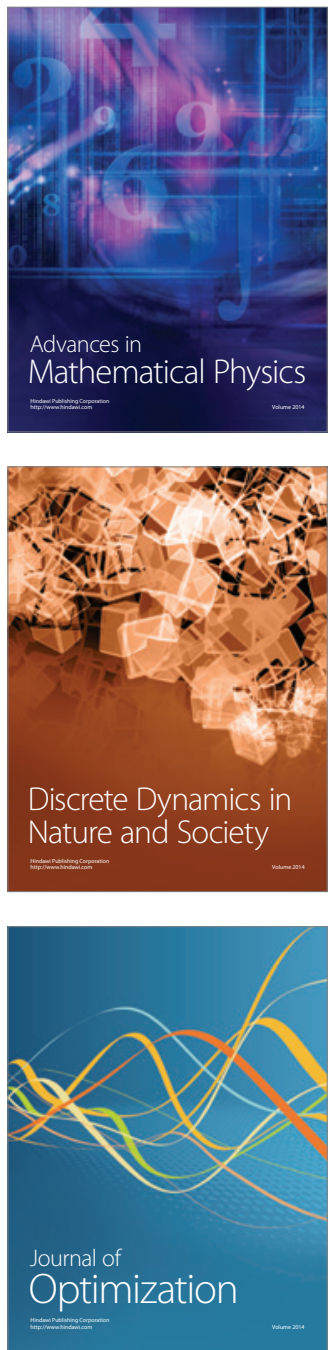